\title{
Reconfigurable curved metasurface for acoustic cloaking and illusion
}

\author{
Shi-Wang Fan ${ }^{1,2}$, Sheng-Dong Zhao ${ }^{1,4}$, Liyun $\mathrm{Cao}^{2}$, Yifan Zhu ${ }^{2}$, A-Li Chen ${ }^{1}$, Yan-Feng Wang ${ }^{1,3}$, Krupali Donda ${ }^{2}$, Yue-Sheng \\ Wang $^{1,3, *}$, Badreddine Assouar ${ }^{2}, *$ \\ ${ }^{1}$ Institute of Engineering Mechanics, Beijing Jiaotong University, Beijing, 100044, China \\ ${ }_{2}^{2}$ Institut Jean Lamour, CNRS, Universite de Lorraine, Nancy, 54000, France \\ ${ }^{3}$ Department of Mechanics, School of Mechanical Engineering, Tianjin University, Tianjin, 300350, China \\ ${ }^{4}$ School of Mathematics and Statistics, Qingdao University, Qingdao, 266071, China \\ *Corresponding authors: yswang@tju.edu.cn \& badreddine.assouar@univ-lorraine.fr
}

\begin{abstract}
A severe limitation of current acoustic metasurfaces remains in their modest tunability to meet multi-frequency requirements and alterable functionalities on demand. Here, a reconfigurable curved acoustic metasurface for acoustic cloaking and illusion is reported. The structure is composed of an array of tunable helical units to break this limitation and realize continuously versatile sound manipulations. We theoretically, numerically and experimentally investigate the channel length represented by the helical depth, which is used to achieve full $2 \pi$ phase shift continuously over the frequency range from 2 to $7 \mathrm{kHz}$. As pragmatic examples, we present by full-wave numerical simulations the concept of a curved metasurface for the continuously tunable acoustic multifunction, including broadband carpet cloaking and ground illusion at a wide working band. Then, we experimentally demonstrate these functionalities by showing an excellent effect to restore the disturbed reflective field from a cloaked object or to mimic an arbitrary shaped ground.
\end{abstract}

Keywords: Acoustic metasurfaces, Curved metasurfaces, Reconfigurability, Carpet cloak, Ground illusion.

\section{Introduction}

Among the various functions of metamaterials, invisibility cloak is one of the most promising applications to effectively control the propagation of classical waves and hide the signature of objects. It is directly related to the original idea of electromagnetic cloaking [1], but has rapidly been extended to other physical fields, including acoustics [2, 3], elastic waves [4,5] and more recently waves in geomaterials [6]. Based on the coordinate transformation technique, waves can, at least theoretically, be smoothly guided around a hidden cylindrical or spherical area and propagate through the cloak without any distortion in the far-field radiation. However, the constitutive parameters of the diffractive cloaking metamaterials contain extreme values and usually challenging to design [7-11]. Therefore, as a special type of cloaking shell, the so-called carpet cloak [7] or ground cloak [8,9] was proposed to hide objects on a flat reflected surface. Due to its finite anisotropy and homogeneity, the carpet cloak has remarkable property of not requiring extreme material parameters for its realization. However, the huge size of these clocking shells are usually comparable with that of the hidden region or even an order of magnitude larger than the objects. This limits its potential applications. 
To get rid of the structural bulky restriction, it recently was reported that metasurfaces with phase discretization $[10,11]$ and their subwavelength thickness feature can replace the conventional metamaterials with transformation method [1-3, 7-9] when it comes to implement efficient carpet cloaking devices. These ultra-thin structures have, indeed, been widely used in various wave field modulations. To be specific, shortly after the development in optics [10], the constructive idea was introduced into acoustics with the purpose of designing diverse subwavelength sound modulatory devices [11]. Now, it is also being gradually applied for elastic waves manipulation [12-14]. The metasurfaces are composed of gradient units based on the generalized Snell's law to manipulate the transmitted or reflected wavefronts in a predefined and unconventional way. That means it does not have to design and modify the anisotropic material parameters like transformation approach does.

The essence of the metasurface carpet cloak is to design an ultrathin structure, which consists of artificial micro-units, by phase discrete treatment, and place it over the concealed objects for restoring the wavefront as if it were reflected from a flat surface. The cloaked target would be then indistinguishable from the reflective field by external detection signals. In optics, the efficient hiding of two-dimensional (2D) and three-dimensional (3D) arbitrarily shaped objects separately in terahertz range [15] and visible light range [16] by metasurface carpet cloaks have been reported. By changing the electric bias of the graphene constituents without varying the optic metasurface geometry, Biswas et al. [17] theoretically demonstrated the versatility of a tunable graphene-based metasurface cloak which is capable of actively switching between regimes of operation. For the acoustic carpet cloaks, several ultrathin gradient metasurfaces were designed to control the local reflected phase and restore the far-field radiation pattern based on Helmholtz resonators [18-20], membrane vibrations [21-23], spiral paths [24], diffraction gratings [25], and so on. These studies show that the metasurface carpet cloak can hide objects with arbitrary shape and size and make them hard to detect acoustically over a wide range of incident angles.

Nevertheless, the Helmholtz cavity and membrane based on resonance mechanism [18-23] will result in a narrow band near a resonant frequency. The spiral path and diffraction grating could be classified as space coiling structure [11,24-27]. Space coiling is a technique where the desired phase shifts can be achieved by the accumulation of the phase along the effective sound path in a labyrinthine-type structure $[26,27]$. However, the conventional space coiling cloaks are typically predefined by the metasurface geometry and cannot be changed once the device was manufactured. This might be a significant limitation when tuning of the device characteristics is essential in actual applications, particularly with the tunable steering of oblique incident detection signals. No investigation on tunable or reconfigurable acoustic metasurfaces for carpet cloaking has been reported up to now. Therefore, it is highly desirable to achieve tunable metasurfaces in which the cloaking characteristics of acoustics can be continually adjusted to broaden and tune the regulation frequency. 
Attempts to this end, little research has been done on the tunable acoustic metasurface [28-35]. By adjusting the orientation of the metasurface with tailored internal losses [28] and changing the distance between two metasurfaces [29, 30], the acoustic metasurfaces of a bilayer configuration were utilized to realize the controllable asymmetric transmission based on Helmholtz resonators. Recently, Ma et al. [31] designed a spatial sound modulator based on an active membrane metasurface and realized it experimentally. The switching mode of the acoustic metasurface can be achieved by switching the DC voltage across an electromagnet attached on individual units. A programmable acoustic metasurface has been reported by Tian et al. [32]. Each unit cell of this metasurface was composed of a straight channel and five shunted Helmholtz resonators, which can be tuned by a fluidic system. For the space coiling metasurface, Zhao et al. [33] designed a continuously tunable acoustic metasurface with individual unit components of a helix screwed inside a cylindrical thin plate for transmitted axial 3D focusing. The idea was then extended to $2 \mathrm{D}$ multifunction switching of a tunable metasurface [34]. The realistic solution to achieve broadband tunability of a flat acoustic metasurface with reflected multifunction was demonstrated numerically and experimentally in this space coiling framework [35]. In the present research, we further enrich and develop the literature [35]. We, indeed, propose an equivalent theoretical model for the helical unit to predict the phase shift response. We then, conduct experimental measurement in a broadband range is to verify our model. The designed tunable units are applied to conceive a curved metasurface to restore or mimic the reflected waveforms.

From the above discussions, the ways to overcome the restriction of fixed and rigid acoustic metasurfaces with single or narrow band are to design a lossy structure or multilayer metasurfaces [28-30], or an independently adjustable unit [31-35] to change the formed geometry. Here, in the present work, a successful concept of a tunable metasurface for carpet cloak is proposed based on a type of helical units using matched screw-and-nut physical mechanism. Our concept is general; the utilization of helical channels [36-40] can lead effectively to low-loss applications in acoustic reflections, while the Helmholtz resonators can be of higher loss due to small sections of their neck [28-30, 32]. We specifically design an adjustable matched helical unit for broadband phase shifts. The equivalent theoretical model, the numerical simulation and experimentally demonstration are performed to get the continuously reflected phase responses. Then it is applied to design the broadband tunable metasurface cloak which can provide the proper phase distribution by adjusting the helical depths to restore reflected waveforms. We furthermore extend the tunability of the curved metasurface to the discussions regarding the wide-angle oblique incidence and acoustic ground illusion, which were rarely mentioned in the previous studies but are very useful for practical applications. In addition, it is easy to achieve and more suitable to construct a metasurface for 3D modulation of acoustic cloaking (see Appendix E). A real-time tunable broadband metasurface cloak could be realized by designing a self-feedback system with programmable automatic control. The 
design concept is shown in Appendix F.

\section{Design principle and structure}

Based on the classical Snell's law, when an incoming wave with an incident angle $\theta_{i}$ impinges onto a flat surface, the reflected angle $\theta_{r}$ will be the same as the incident angle (termed as global incident and reflected angle $\theta_{i}^{g}=\theta_{r}^{g}$ ). However, if a metasurface is applied to cover the surface, the reflected wave may propagate in any desired direction $[10,11]$.
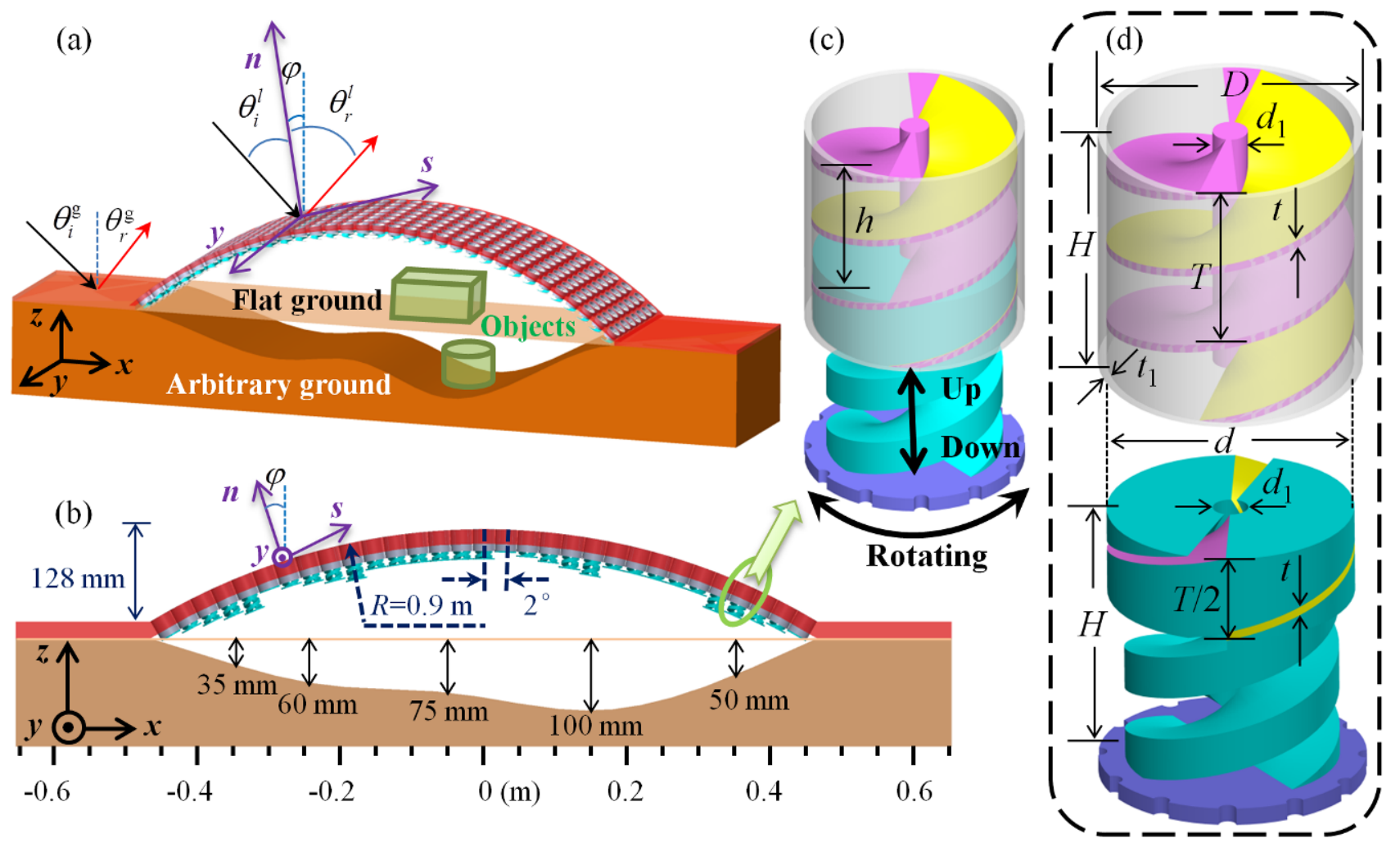

FIG. 1. A 3D schematic view and working principle of an arc metasurface carpet cloak based on a tunable

matched helical units. (a) The schematic diagram of the generalized Snell's law for the wavefront restoration of the curved metasurface with infinite length in $y$-axis direction. It will be used to mimic two types of surfaces, including flat and arbitrary shaped ground. (b) Schematic view of a cross section of the metasurface cloak with distributed helical units. The metasurface is an arc-shaped plate with radius of $R=0.9 \mathrm{~m}$ and the arc central angle of $60^{\circ}$. Thirty-one helical units are uniformly arranged on its surface with step of $2^{\circ}$. (c) The cylindrical unit of the tunable cloak. The depth of the helical channel $h$ is the tunable parameter of the system by rotating a matched screw into a helical channel from bottom to top and allows the acoustical phase response to be continuously controlled. (d) The cylindrical geometric details of the helical channel and matched screw.

To illustrate our design strategy of the cloaking structure, we consider an arbitrary scattering surface $z_{m}=z_{m}(x)$ as shown in Figs. 1(a). Treating each point on the curved surface locally as an arc plane with a length $d s$, it has a radius $R$ and a tilt angle $\varphi$ with respect to the vertical direction. We suppose that the counterclockwise is positive. The new local incident angle $\left(\theta_{i}^{l}\right)$ will no longer equal 
to the local reflected angle $\left(\theta_{r}^{l}\right)$ and be changed along the curved surface, that is, $\theta_{i}^{l}=\theta_{i}^{g}-\varphi$. When waves impinge on the arbitrary-shaped metasurface, the relation of the incident and reflected waves is given in local coordinates $(s-y-n)$ by the generalized Snell's law of reflection as

$$
k_{0}\left(\sin \theta_{r}^{l}-\sin \theta_{i}^{l}\right)=-d \phi(s) / d s,
$$

where $\phi(s)$ is the reflected phase delay or compensation provided by the metasurface; and $k_{0}=2 \pi / \lambda$ is the wave number in the medium air. $\lambda=c_{0} / f$ is the wavelength with $f$ being the frequency and $c_{0}=343 \mathrm{~m} / \mathrm{s}$ the wave velocity. Eq. (1) implies that the reflected sound direction or wavefront shape can be freely steered by presupposing the suitable phase shift $\phi(s)$ along the curved surface. Due to the tilt angle has the geometrical relationship as $\varphi=\theta_{i}^{g}-\theta_{i}^{l}$, the local reflected angles of specular reflection for carpet cloaking can always be expressed as $\theta_{r}^{l}=\theta_{r}^{g}+\varphi=\theta_{i}^{g}+\varphi=2 \theta_{i}^{g}-\theta_{i}^{l}$. In this case, Eq. (1) can be written as

$$
d \phi(s) / d s=-k_{0}\left[\sin \left(2 \theta_{i}^{g}-\theta_{i}^{l}\right)-\sin \left(\theta_{i}^{l}\right)\right]=-2 k_{0} \cos \theta_{i}^{g} \sin \varphi,
$$

which, after integration with consideration of $d s=-R d \varphi$, yields the phase shift: $\phi=-2 R k_{0} \cos \theta_{i}^{g} \cos \varphi+C$. Then according to the relation between global and local coordinates, that is, $(x, z)=(R \sin \varphi, R \cos \varphi)$, the needed phase shift for the carpet cloaking of the curved metasurface is given by

$$
\phi(x)=-2 k_{0} z_{m}(x) \cos \theta_{i}^{g}+C
$$

where $C$ is an arbitrary constant and is chosen from the known phase of the flat ground. It can be found from Eq. (3) that for the specular reflection of a flat surface without height difference $z_{m}(x)=0$, the phase distribution is a constant $C$ as it should be. In addition, the required phase distribution is only related to the global incident angle $\theta_{i}^{g}$ and the surface height $z_{m}(x)$ at a given frequency. It is noticeable that the derived process of Eq. (3) is an accurate and general expression for carpet cloaking, which does not require an approximation like some previous literatures[20, 41, 42]. Therefore, once an object is covered with a curved metasurface which satisfies Eq. (3), no explorer can distinguish it from a flat surface. This is the basic idea of the design for a metasurface carpet cloak.

In addition, we suppose that an arbitrary ground surface is parameterized by a function of $z_{g}=z_{g}(x)$, as shown in the bottom of Fig. 1(b). The reflection of the waves from such a surface is governed by the standard Snell's law of reflection. Similarly to the carpet cloaking, we can design the required phase distribution by a metasurface to mimic the reflected pattern of an arbitrary ground just simply calculating the surface height difference. Then, the metasurface can exhibit the same reflecting performance as the ground surface, which is known as ground illusion [17, 20]. Consequently, no explorer can distinguish some objects between the curved metasurface and the arbitrary ground. 
As illustrated in Fig. 1(b), the curved metasurface is described by an arc-shaped equation of $(x-a)^{2}+(z-b)^{2}=R^{2}$. The coordinates of the circle center $(a, b)$ is equal to $\left(0,-R \cos 31^{\circ}\right)$ which makes the vertical coordinate of the ground zero. $R=0.9 \mathrm{~m}$ is the radius of the arc-shaped metasurface which is formed by a lattice distribution of the unit cells with angular intervals of $2^{\circ}$. Then, the height profile of the ground is mapped out by parameterizing an arbitrary curve. We consider a plane wave incident to the metasurface and set the initial phase of the ground plane to be zero. Hence, according to the coordinates of the arc-shaped surface, the phase shift required to achieve the carpet cloak and ground illusion can be obtained as

$$
\begin{aligned}
& \phi(x)=-4 \pi f\left[z_{m}(x)-z_{g}(x)\right] \cos \theta_{i}^{g} / c_{0}, \\
& x^{2}+\left(z_{m}+R \cos 31^{\circ}\right)^{2}=R^{2}, z_{g}=z_{g}(x), \\
& -R \sin \varphi \leq x \leq R \sin \varphi, \varphi \in\left[0,30^{\circ}\right], \Delta \varphi=2^{\circ} .
\end{aligned}
$$

Therefore, having the appropriate phase distribution as dictated by Eq. (4), we can hide an arbitrary object by making it look like a flat plane or an arbitrary desired ground using the arc-shaped metasurface cloak.

The individual unit of the helical-structured metasurface we propose consists of a matched screw rotated into a helical channel from bottom to top, as shown in Fig. 1(c). Based on the matched screw-and-nut mechanism [33-35], when the matched screw is tuned by rotating, the channel length characterized by the helical depth $(h)$ will be changed. The scalar air-borne sound will propagate freely into the unit along the helical paths and is reflected from the upper surfaces of the matched screw. The reflected phase shift can be then varied by changing the helical depth. In this way, the present flexible tunable units constitute a metasurface which can compensate or delay the phase shift to reshape the reflected wavefront through the change of the helical depth. The latter is the only tunable parameter of the system.

As illustrated in the top of Fig. 1(d), the helical channel is constructed by placing and fixing a cylindrical helix into a hollow-cylinder. The cylindrical helix is composed of two rectangular blades spiraling around a central slender column. The detailed geometry parameters of the unit can be defined as: the total height is $H=30 \mathrm{~mm}$, the cylinder diameter is $D=30 \mathrm{~mm}$, the thread lead is $T=20 \mathrm{~mm}$, the column diameter is $d_{1}=4 \mathrm{~mm}$, the blade thickness is $t=1 \mathrm{~mm}$ and the wall thickness of the hollow cylinder is $t_{1}=1 \mathrm{~mm}$. The diameter of the penetrating hole is $d=D-2 t_{1}=28 \mathrm{~mm}$. In the bottom of Fig. 1(d), the rest of the cylinder removing the cylindrical helix constitutes the matched screw of which the inner and outer diameters are the same as the diameters of the hole and slender column, respectively. We decrease the external diameter and increase the internal diameter on its lower part to reduce rotational friction. To facilitate the rotation and connection of a motor for mechanical control (see Appendix F), we attach two matched helixes together with a thin sheet and set up a ring gear by distributing grooves on its edge. 


\section{Analyses of phase shifts for the helical unit}

In order to achieve a tunable design, we must obtain the continuous relation between the phase shift $(\varphi)$ and the helical depth $(h)$ at a broadband frequency $(f)$ domain. To this end, we demonstrate the phase shifts response to the frequency and depth for the proposed helical unit. By using the transfer matrix method, the equivalent propagation path of the theoretical model is obtained to calculate the phase shifts. The numerical simulation and experimental measurement for the phase shifts will be performed to verify the analytical results and to compare to each other.
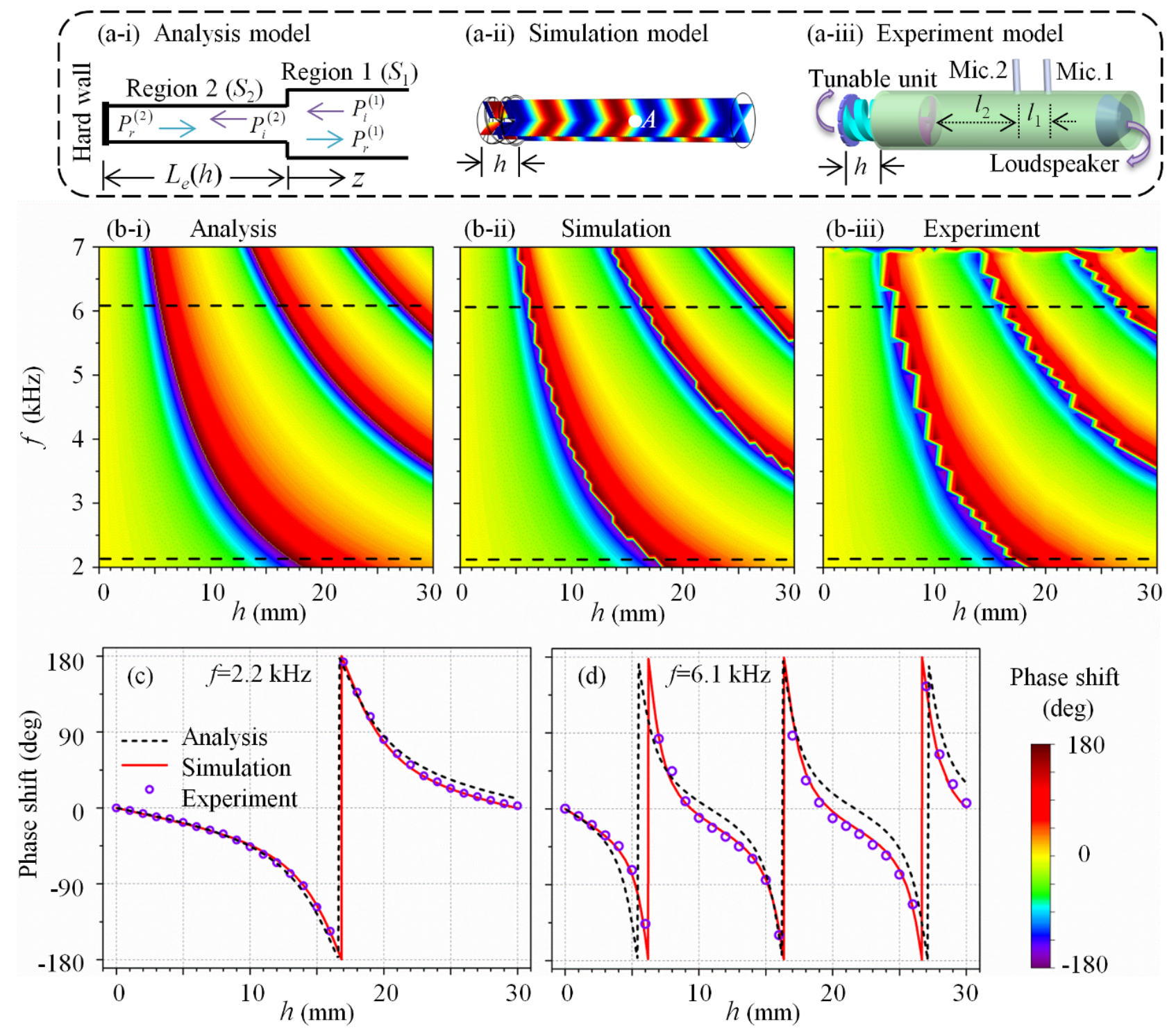

FIG. 2. Theoretical, numerical and experimental phase shift response to the frequency domain and the helical depth for the tunable cylindrical unit. (a) The schematic of the (i) analytical structure, (ii) simulated model and (iii) measured acoustic impedance tube. (b) The corresponding phase shift of the tunable unit. (c-d) The cut lines of (b) at the frequencies of $2.2 \mathrm{kHz}$ and $6.1 \mathrm{kHz}$ showing the variation of the phase degree with the helical depth. 


\section{A. Theoretical model analysis}

Because sound is a scalar wave, the acoustics can propagate freely inside channels. Then, the space-coiling spiral channels of the unit can be represented as an equivalent straight channel. The boundary end of the channel is a hard wall. The unit-cell is divided into two regions with different cross sections, as illustrated in Fig. 2(a-i). Then ignoring the time harmonic factor, we can express the acoustic pressures as:

$$
P_{i}^{(j)}=A_{j} \exp (i k z), \quad P_{r}^{(j)}=B_{j} \exp (-i k z),
$$

where $P_{i}^{(i)}$ and $P_{r}^{(j)} \quad(j=1,2)$ denote the incident and reflected waves in the $j$ th region; and $A_{j}$ and $B_{j}$ are the corresponding amplitudes. The equivalent length of region 2 is $L_{e}$, which is directly related to the equivalent diameter $D_{e}$ and the channel diameter $d$. Since waves at different frequencies have different wavelengths, the equivalent diameter in the spiral channel will change with the incident frequency, $D_{e}=e d$. The equivalent parameter $e=e(f)$ dependent on the incident frequency can be obtained after the numerical fitting between the lengths of the helical path in the unit and the straight path in free-space based on the unit helicity [36, 39, 43]. For more details of the equivalent parameter, we refer to Appendix A.

The continuity of pressure and volume velocity should be satisfied on the inter boundary between region 1 and region 2 at $z=0$. The left boundary is a hard wall at $z=-L_{e}(h)$. Here, the total pressure is twice of the incident pressure; and the resultant velocity is zero. Therefore, the boundary conditions at the two interfaces can be expressed as

$$
Q_{1}\left[\begin{array}{l}
A_{1} \\
B_{1}
\end{array}\right]=Q_{2}\left[\begin{array}{l}
A_{2} \\
B_{2}
\end{array}\right], \quad Q_{3}\left[\begin{array}{l}
A_{2} \\
B_{2}
\end{array}\right]=Q_{4}\left[\begin{array}{c}
A_{2} \\
0
\end{array}\right],
$$

with the coefficient matrices being

$$
\begin{aligned}
Q_{1} & =\left[\begin{array}{cc}
1 & 1 \\
S_{1} & -S_{1}
\end{array}\right], \quad Q_{2}=\left[\begin{array}{cc}
1 & 1 \\
S_{2} & -S_{2}
\end{array}\right], \\
Q_{3} & =\left[\begin{array}{cc}
\exp \left(-i k L_{e}\right) & \exp \left(i k L_{e}\right) \\
S_{2} \exp \left(-i k L_{e}\right) & -S_{2} \exp \left(i k L_{e}\right)
\end{array}\right], \quad Q_{4}=\left[\begin{array}{cc}
2 \exp \left(-i k L_{e}\right) & 0 \\
0 & 0
\end{array}\right],
\end{aligned}
$$

where $S_{1}$ and $S_{2}$ are the cross-section areas of regions 1 and 2, respectively. According to the geometrical relation of helical channel, the following parameters can be obtained: 


$$
\begin{aligned}
& S_{1}=\pi(d / 2)^{2}, S_{2}=(T / 2-t)\left(d-d_{1}\right) \cos \alpha, \\
& L_{d}=\sqrt{(\pi d)^{2}+T^{2}}, l_{d}=\sqrt{\left(\pi d_{1}\right)^{2}+T^{2}}, \\
& L_{e}=h \sqrt{\left(\pi D_{e}\right)^{2}+T^{2}} / T, \quad \alpha=1-\left(L_{d}-l_{d}\right) /\left[\pi\left(d-d_{1}\right)\right], \\
& e=1.385 \times 10^{-9} f^{2}+7.71 \times 10^{-6} f+0.4619,
\end{aligned}
$$

in which $\alpha$ is the rising angle of the spiral blade; and $L_{d}$ and $l_{d}$ are the spiral length of single helix at the external and internal boundary of the helical channel, respectively.

We finally obtain the relation of the incident and reflected wave fields for the unit based on the transfer matrix method as

$$
\left[\begin{array}{l}
A_{1} \\
B_{1}
\end{array}\right]=Q\left[\begin{array}{c}
A_{2} \\
0
\end{array}\right]
$$

where combining Eqs. (6), (7) and (9), the total transfer matrix can be expressed as

$$
Q=\left[\begin{array}{ll}
t_{11} & t_{12} \\
t_{21} & t_{22}
\end{array}\right]=Q_{1}^{-1} Q_{2} Q_{3}^{-1} Q_{4},
$$

The normalized amplitude and phase shift of reflection $(A$ and $\phi)$ can be calculated by

$$
A=\left|\frac{A_{r}}{A_{i}}\right|=\left|\frac{t_{21}}{t_{11}}\right|, \quad \phi=\arg \left(\frac{A_{r}}{A_{i}}\right)=\arg \left(\frac{t_{21}}{t_{11}}\right) .
$$

Based on Matlab programming calculation, it can be found from Eq. (11) that the reflected amplitude is always equal to 1 . This means total reflection from the helical structure without any energy loss. Then, the phase shift responses varying with the incident frequency $(f)$ and helical depth $(h)$ for the theoretical model is calculated and illustrated in Fig. 2 (b-i). Note that, under the geometry parameters of our design, the phase shifts of the reflected waves cover the full $2 \pi$ range at the frequencies above $2 \mathrm{kHz}$. For the upper limit of the operating frequency, logically speaking, the bandwidth can be extended to the entire subwavelength range due to the continuous tunability of the helical unit. However, the relative size of the incident wavelength and the unit diameter are very important factors that affect the performance of the wavefront manipulations because of diffraction effect (see Appendix C). In addition, the reflected phase shift covers multiple $2 \pi$ spans with several mutation positions at high frequencies, which means a high sensitivity and inconvenient adjustment. The numerical tests show that the appropriate upper limit of the operating frequency should be 7 $\mathrm{kHz}$.

\section{B. Finite element simulations}

To obtain the numerical relation between the phase shift and the helical depth at a continuous 
frequency domain, a finite element model for the unit is developed in Fig. 2(a-ii) based on the commercial software COMSOL Multiphysics 5.3. The sound medium is air. The free surfaces of the solid structures are set to sound hard boundaries. The background pressure field is used to excite the incident plane waves. The plane wave radiation boundary condition is imposed on the incident boundary to absorb the reflected waves. For the cylindrical incident field, the sound hard condition is assumed on the side boundaries. The calculated reflected phase shift is exported at the identical arbitrary observation point $A$ which has a distance of $66 \mathrm{~mm}$ from the unit surface in our simulation model. Because the key factor is not the phase value itself but the relative phase between the different helical depths, in principle the phase extracting position can be chosen arbitrarily by avoiding the influence of the boundary effect $[11,33,35]$. In addition, the phase shifts varying with the helical depths at the incident frequencies will start from zero at the chosen observation point. This will facilitate the comparison with the above analytical results and the following experimental results. The tunable helical depth ranges from $0 \mathrm{~mm}$ to $30 \mathrm{~mm}$ by step of $0.5 \mathrm{~mm}$. We sweep the frequency by a step of $100 \mathrm{~Hz}$ at every given helical depth. Then, the phase shift responses varying with the incident frequency $(f)$ and helical depth $(h)$ for the simulation model are computed and shown in Fig. 2 (b-ii). Note that, as acoustic waves propagate through the helical channels of the tunable unit, the thermoviscous loss effects could influence the reflected phase and amplitude back from the curved metasurface, especially when considering the high frequency of the operation. We use the thermoviscous acoustics model to calculate the phase shift and reflected amplitude of the acoustic wave from the tunable unit. And we also present the acoustic pressure field distribution of the illusion metasurface at a high frequency $(6.1 \mathrm{kHz})$ using the thermoviscous acoustics model. The results indicate that the influences of the thermoviscous loss on the tunable acoustic performances are very weak and could be ignored, see Appendix D for details. Thus, in this work, we do not consider the thermoviscous loss effects in the following simulations.

\section{Experimental measurement}

By using the Brüel \& Kjær impedance tube kit (type 4206, small tube), shown in Fig. 2(a-iii), the reflected factor $\operatorname{Re}=A \exp (i \varphi)$ can be obtained based on the transfer function method as follows

$$
R e=\frac{P_{12}-\exp \left(-i k_{0} l_{1}\right)}{\exp \left(i k_{0} l_{1}\right)-P_{12}} \exp \left[2 i k_{0}\left(l_{1}+l_{2}\right)\right],
$$

where $P_{12}=P_{2} / P_{1}$ is the transfer function between the acoustic pressure measured by the microphones (Mic.1 and Mic.2, Brüel \& Kjær type 4187), and processed by the Brüel \& Kjær PULSE Multi-analyzer system. Then, the reflected amplitude and phase can be directly calculated from $A=$ $|R e|$ and $\phi=\arg (R e)$.

As previously mentioned, the phase shift is a relative value. However, the phase difference between the different helical depths must meet a certain value independent of the measuring point. 
Then the tunable unit is placed into the impedance tube with staying the fixed distance $l_{2}$ during the unit adjustment to sweep frequency from 2 to $7 \mathrm{kHz}$. For every incident frequency, each data at different helical depths $\left(h_{i}\right)$ is subtracted from the initial phase value $\left(h_{0}=0\right)$. This process sets the phase shift to be with zero initial value. Then, it can be compared with theoretical model and numerical simulation, as shown in Fig. 2(b-iii). It should be mentioned that the Brüel \& Kjær impedance small tube set-up can measure parameters in the frequency range from $500 \mathrm{~Hz}$ to $6.4 \mathrm{kHz}$, and the accurate phase at a higher frequency cannot be obtained by this experiment. Therefore, the phase information shows a little chaos as it approaches $7 \mathrm{kHz}$. Even so, the theoretical model and numerical simulation can still be validated by the data below $7 \mathrm{kHz}$.

As shown in Fig. 2(b), the theoretical, numerical and experimental results are consistent with each other in terms of the number across full $2 \pi$ ranges and the phase shift trend. For a more intuitive quantitative comparison, the cut lines of the phase shift at the frequencies of 2.2 and $6.1 \mathrm{kHz}$ [dashed lines in Fig. 2(b)] are plotted in Figs. 2(c) and 2(d). One can observe that the three models yield almost the same phase shifts at the same frequency and helical depth. Therefore, these results show that the broadband tunable unit manipulating the reflected acoustic waves can be achieved by continuously adjusting the helical depth.

\section{Functions and results}

The developed tunable subwavelength unit has the merit of continuously modulating the reflected phase shift covering a full $2 \pi$ span by gradually changing the helical depth with rotating a matched screw. This advantage establishes the foundation for dynamically manipulating acoustic waves at a broadband frequency range by using our helical metasurface. Multiple functions can be easily realized through the reconfiguration of only one metasurface, whereas the traditional metasurfaces with fixed configurations require costly and time/labor-consuming redesigning and fabrication [32-35]. Next, we first will exhibit two useful dynamic functions, including carpet cloak and ground illusion, by full-wave numerical simulations. Then, the corresponding experimental measurements will be carried out to demonstrate the hidden and illusive performances using a fabricated metasurface.

\section{A. Full-wave numerical simulations}

To show the performance of the designed curved cloak [see Figs. 1(b)], full-wave simulations of the reflected fields are carried out with COMSOL at the different target frequencies. A background pressure field are utilized to excite the monochromatic incident waves which makes it easier to derive the reflected field directly. The upper, left and right boundaries of the calculation domain are bounded by plane wave radiation conditions which allows the waves to propagate out from the domain without reflections. All the other free surfaces of the unit and waveguide structures are set to 
sound hard boundaries. Then the reflected performances can be calculated according to the configurations of the unit helical depth on the metasurface.

\section{Carpet cloak}

Due to the existence of the height difference between the curved cloak and the flat ground $\left[z_{g}(x)=0\right]$, the propagation of the reflected waves from the arc surface needs to be delayed. We first consider a plane wave normally incident to the ground and the metasurface, that is, $\theta_{i}^{g}=0$. The first formula of Eq. (4) can be expressed as $\phi(x)=-4 \pi f z_{m}(x) / c_{0}$; then the required deferred phase of each unit can be calculated from this formula according to the vertical coordinates of the unit center on its upper surface. Once the needed phase degrees are known, we can obtain the corresponding helical depth from Fig. 2. For a given incident sound frequency in the subwavelength range, e. g. $f=2.2 \mathrm{kHz}$, the first unit on the left is rotated by $\varphi_{1}=30^{\circ}$ and positioned at the location of $x_{1}=-R \sin \varphi_{1}=-0.45 \mathrm{~m}$ and $z\left(x_{1}\right)=\operatorname{sqrt}\left(R^{2}-x_{1}^{2}\right)-R \sin 31^{\circ}=7.9 \mathrm{~mm}$. Then the needed phase delay can be obtained as $\phi_{1}\left(x_{1}\right)=-4 \pi f z_{m}\left(x_{1}\right) / c_{0}=-36.8^{\circ}$. Therefore, the first helical depth should be $h_{1}=8.6 \mathrm{~mm}$ according to Fig. 2(c). Similarly, we can find the associated depth for each unit of the metasurface from Fig. 2 to achieve multifunctions at different frequencies. For a sake of clarity and convenience, the required depth value is ranked to $h_{i}(\mathrm{~mm})(i=1,2, \ldots, 31)$ from left to right. The results are given clearly in Table I in Appendix B. 


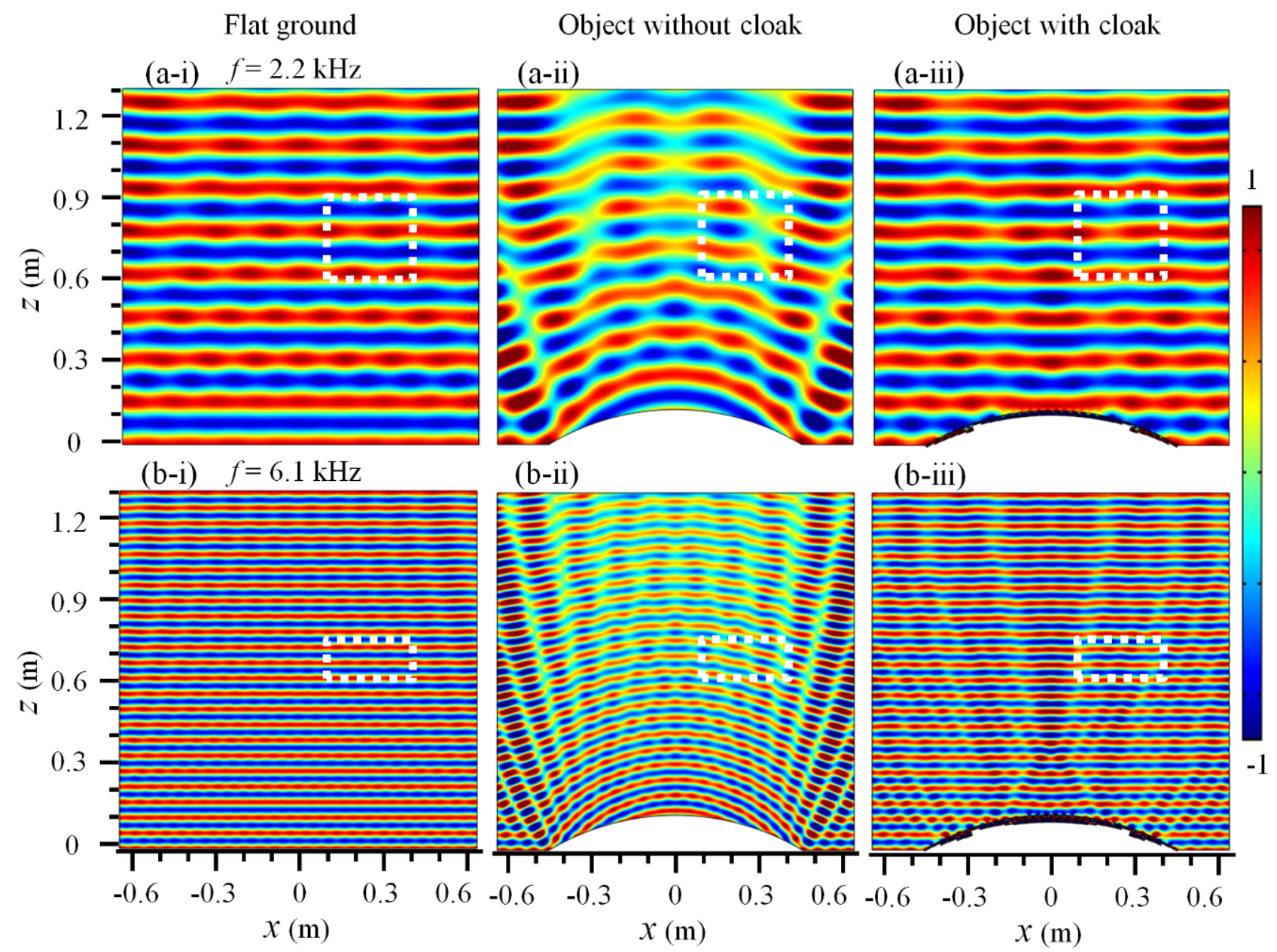

FIG. 3. Full-wave simulated scattering acoustic pressure fields for carpet cloaking. The reflected fields in the $x z$-plane in three cases: (i) on a flat ground, (ii) on an uncloaked object (arc-shaped bump) and (iii) on the object with the cloak exposed to the normally incident plane wave at frequency of $2.2 \mathrm{kHz}$ (a) or $6.1 \mathrm{kHz}$ (b). The white dashed boxes indicate the regions within which the measurement will be performed in the next experimental section.

By employing this helical depth, the reflected acoustic pressure fields are simulated and illustrated in Fig. 3 for the cases when a monochromatic wave impinges normally on (i) the flat ground, (ii) the uncloaked object and (iii) the object covered by the metasurface cloak. The reflected fields for these three cases at the target frequency of $f=2.2 \mathrm{kHz}$ are illustrated in Fig. 3(a). As it can be seen in Fig. 3(a-i), when a sound wave hits the flat ground, it undoubtedly returns from its original path. However, the sound field is significantly distorted due to the presence of the object when no cloaking treatment is applied in Fig. 3(a-ii). Such a strong contrast between the central region and its surrounding implies that the object can be easily detected by scanning the disturbed field. In comparison, covering the object with the designed metasurface restores the original sound field successfully, i.e., the wavefronts are parallel to the horizontal surface, which is almost the same as the reflection by a flat ground, as shown in Fig. 3(a-iii). Hence, it can make the object completely 
hidden behind the arc metasurface cloak at the frequency of $2.2 \mathrm{kHz}$.

In practical applications for carpet cloaking, a signal used for detection may always be changed on different probe. Once waves with another frequency are incident to the object, an entirely new set-up of phase delay configuration needs to be provided by the metasurface, whereas the traditional structures with fixed configurations will not meet the cloaking requirements using only one metasurface. Thanks to the continuously tunable properties of the helical unit, the metasurface designed in this paper can be easily engineered by conveniently reconfiguring the needed phase shift. That is to say, any frequency can be set to the target one in subwavelength range. As an example, Fig. 3(b) shows the contrast of the three cases at the frequency of $6.1 \mathrm{kHz}$. The numerical reflected fields on the flat ground in Fig. 3(b-i) and the cloaked object in Fig. 3(b-iii) are consistent with each other in terms of the horizontal wavefronts by comparing to the uncloaked object in Fig. 3(b-ii). These results reveal that the capability of the metasurface on hiding the object is remarkable over a wide target frequency range. Therefore, a broadband acoustic carpet cloaking can be achieved by the tunable curved metasurface for normal plane wave incidence.

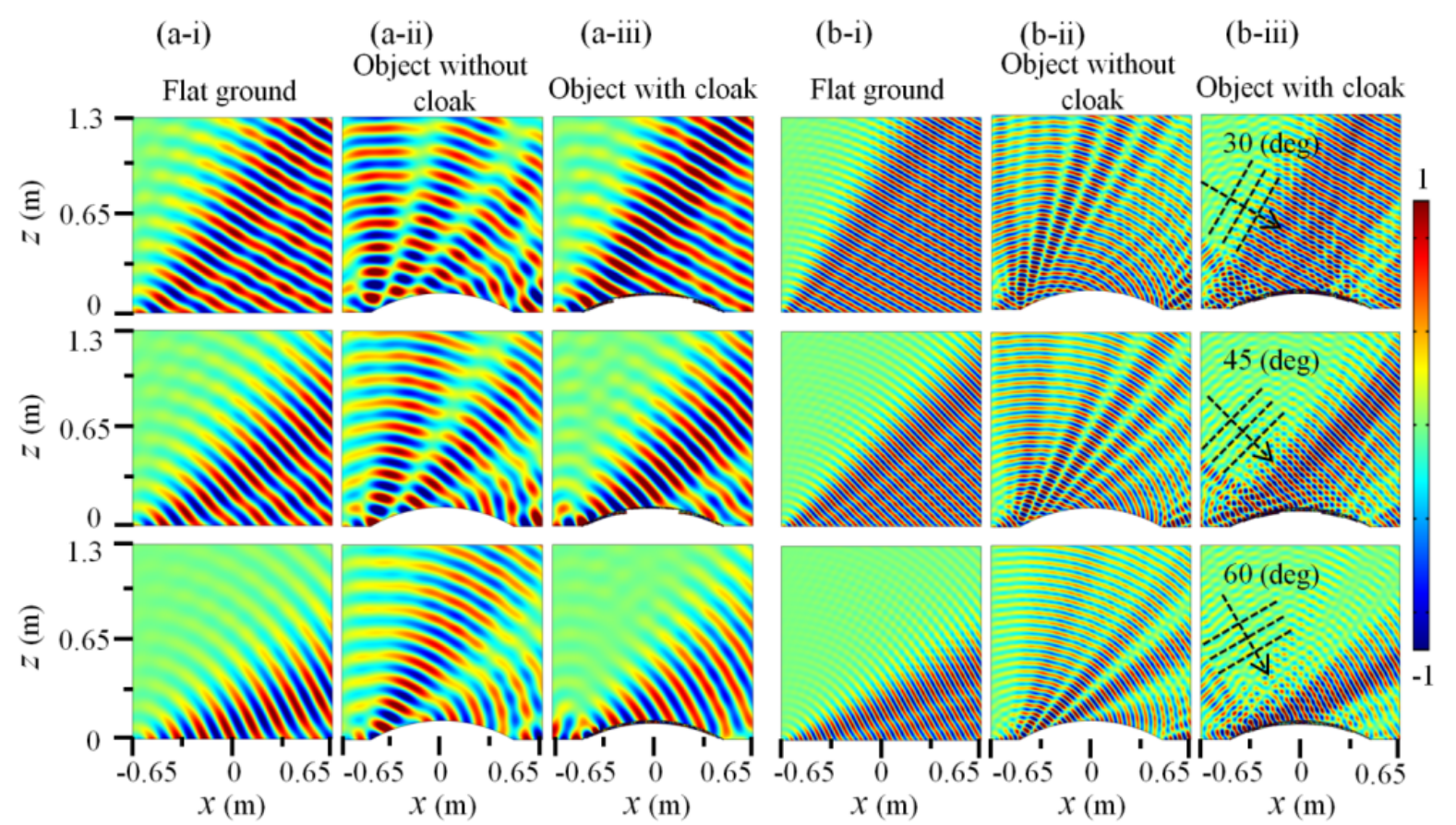

FIG. 4. The simulated scattering pressure fields for oblique incidence. The reflected fields in the $x z$-plane in three cases: (i) on a flat ground, (ii) on an uncloaked object (arc-shaped bump) and (iii) on the object with the cloak exposed to the $30^{\circ}$ (upper row), $45^{\circ}$ (middle row) and $60^{\circ}$ (bottom row) impinging acoustic waves at the target frequency of $2.2 \mathrm{kHz}$ (a) or $6.1 \mathrm{kHz}$ (b). The black dashed lines and arrows set in (b-iii) indicate the incident plane wavefronts and directions and also apply in (a-iii). For all cases in (a-iii) and (b-iii), the angle of reflection from the object with the cloak is designed to be equal to the incident angle like a specular reflection.

The proposed metasurface displays a broadband tunability for normally incident plane waves. However, when a detection signal is sent and received at different locations, that is, multi-base 
station transmitter and receiver devices, the curved metasurface will be required to provide specular reflection at multiple incident angles to conceal objects. By observing its governing equation, i.e. Eq. (4), the cloak could provide hiding performance under oblique incidences. When waves impinge obliquely from upper left with the global incident angle $\theta_{i}^{g}=30^{\circ}$ at the frequency of $f=2.2 \mathrm{kHz}$, the required phase degrees are thereupon determined by the unit center coordinate following Eq. (4) as $\phi(x)=-4 \pi f z_{m}(x) \cos \theta_{i}^{g} / c_{0}=-22.22 \pi z_{m}(x)$ rad. Likewise, by considering the relation between the phase shift and the helical depth in Fig. 2(c), the depth for each unit of the metasurface is obtained as shown in Table I of Appendix B. By employing this helical depth distribution, the reflected acoustic pressure fields of (i) the flat ground, (ii) the uncloaked object and (iii) the object with the cloak are presented in upper row of Fig. 4(a). One can clearly observe that the selected helical depths can indeed guarantee a satisfactory acoustic reflection at $30^{\circ}$ at the frequency of $2.2 \mathrm{kHz}$. Subsequently, the reflected fields for some other suitable depth values of the specular reflection at the angles of $45^{\circ}$ and $60^{\circ}$ are plotted in the middle and bottom rows of Fig. 4(a), respectively. Moreover, in order to fully demonstrate tunability, comparisons of the fields for different incidence angles $\left(30^{\circ}, 45^{\circ}\right.$ and $60^{\circ}$ ) at the frequency of $6.1 \mathrm{kHz}$ are shown in Fig. 4(b). All results indicate that the performance of the metasurface cloak is good enough in a wide incident angle range and a broadband frequency range. In principle, since the invisibility is achieved via symmetrical local phase adjustments, the designed cloak is expected to work well when waves are coming from the other side (upper right). Note that the slight discrepancies between the reflected fields in the cases with ground and the cloaked object may be due to the discretization of the phase shift when building the metasurface cloak.

\section{Ground illusion}

The abovementioned metasurface can be used as a carpet cloak to manipulate the scattered field from an object, which is placed over the object to mimic a flat ground. This statement also could be interpreted as an illusion for the flat ground reflection. With this in mind, let us suppose an arbitrarily shaped convex or concave ground is covered by the designed tunable metasurface for the ground illusions. Eq. (4) gives the phase required to be applied along a metasurface $z_{1}=z_{m}(x)$ to mimic the reflection pattern of another surface, $z_{2}=z_{g}(x)$.

A parameterized covered ground surface with multiple bumps and dents is chosen, whose width is set to the same size as the metasurface $(0.9 \mathrm{~m})$ and its height profile is mapped out in Fig. 1(b). We first consider the convex ground which is formed by symmetry of the concave shape with respect to the $x$-axis and impinged by a plane wave normally incident at the frequency of $f=2.2 \mathrm{kHz}$. Then the required phase distribution is determined by the surface height difference by following Eq. (4) as $\phi(x)=-4 \pi f\left[z_{m}(x)^{-} z_{g}(x)\right] / c_{0}=-25.66 \pi\left[z_{m}(x)^{-} z_{g}(x)\right] \mathrm{rad}$. The depth for each unit of the metasurface is obtained according to Fig. 2(c) as shown in Table II (see Appendix B). 


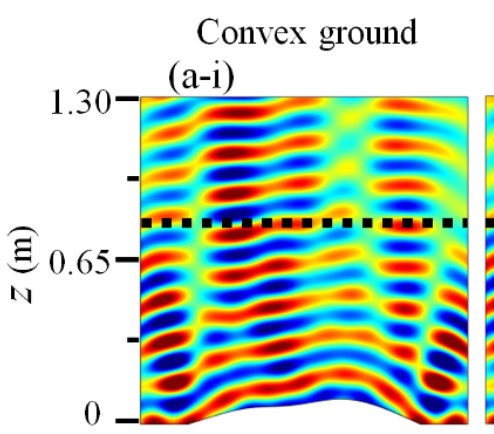

Illusion with cloak (a-ii)
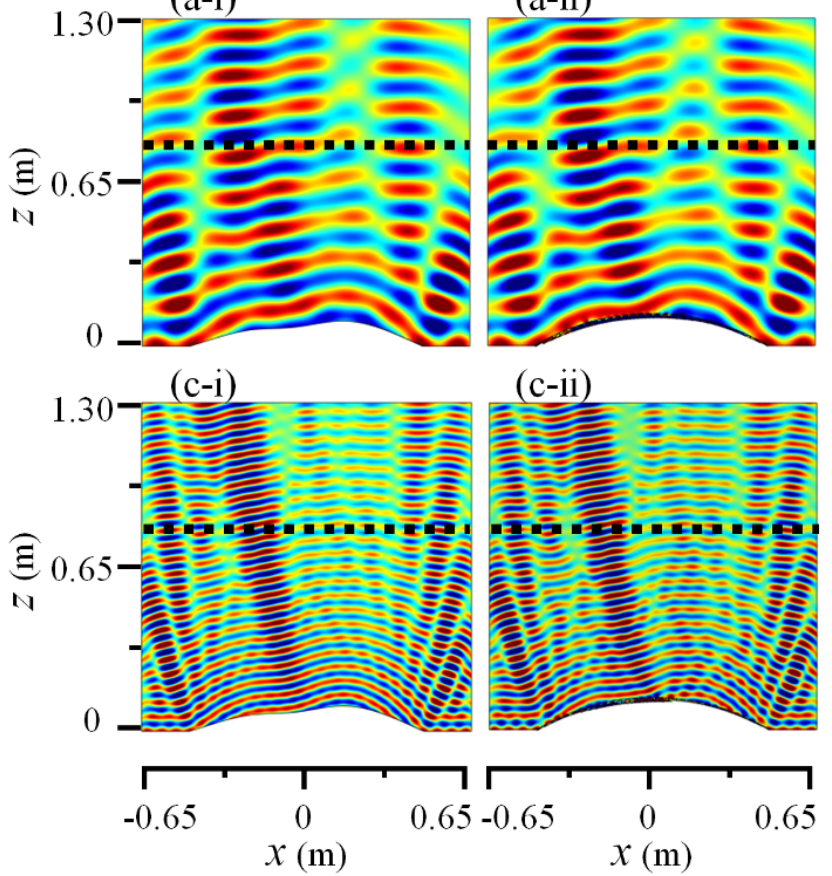

(c-ii)

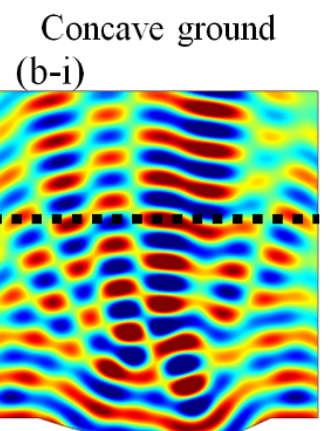

Illusion with cloak (b-ii)

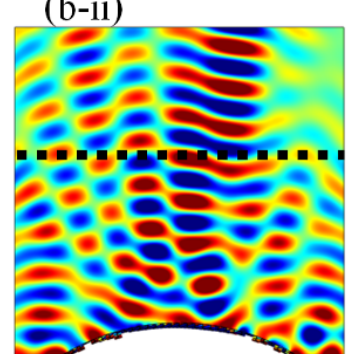

(d-i)

(d-ii)
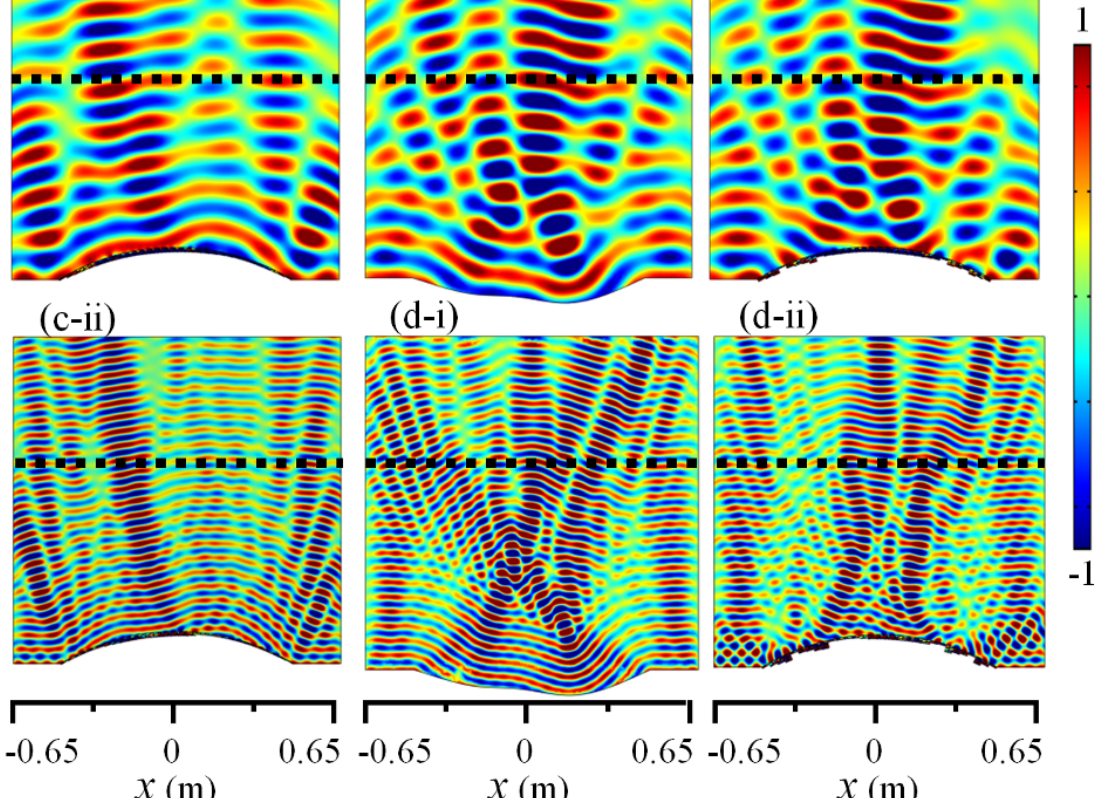

FIG. 5. Full-wave simulated scattering acoustic pressure fields for ground illusion. The reflected fields of (i) a curved arbitrary ground and (ii) the ground illusion with the cloak exposed to the normally incident plane waves at the frequencies of $2.2 \mathrm{kHz}(\mathrm{a}-\mathrm{b})$ and $6.1 \mathrm{kHz}(\mathrm{c}-\mathrm{d})$. The illusion ground is a convex shape in (a) and (c), as well as a concave shape in (b) and (d). The black dashed lines indicate the positions where the measurement will be performed in the next experimental section.

By utilizing this depth values, the reflected fields of (i) the bare ground and (ii) the ground illusion with the cloak are presented in Fig. 5(a). When some objects on the curved ground are covered with the designed metasurface, the scattering pattern becomes similar to that of the bare ground, which would make the arc metasurface appear to be an arbitrarily shaped ground to an external observer. To give one more general illusion in terms of what a greater space the cloak can conceal, the fields plotted for the corresponding concave ground are shown in Fig. 5(b). In addition, for comparison the fields at the frequency of $6.1 \mathrm{kHz}$ for ground illusion of the (i) convex shape and (ii) concave shape are simulated and shown in Figs. 5(c) and 5(d). All comparable performances confirm that there is a good agreement in the far fields between the metasurface structure and the arbitrary ground, especially at the low frequencies.

\section{B. Experimental setups and measurements}

In order to evaluate the validity of the proposed metasurface for cloaking and illusion, we perform the acoustic measurements and analyses in this section. Fig. 6(a) shows the schematic diagram of the experimental setup. The components of the helical unit are fabricated via a 3D printer (PILOT 450, UnionTech) with enough precision $(0.1 \mathrm{~mm})$ to meet the requirement of the proposed 
designs [see Figs. 6(b) and 6(c)]. Thirty-one units are used in the experiment. The arc frame $(1.3 \mathrm{~m} \times 32 \mathrm{~mm} \times 20 \mathrm{~mm}$, radius $R=0.9 \mathrm{~m})$ of the metasurface is fabricated through the mechanical processing with 31 penetrating holes (diameter of $30 \mathrm{~mm}$ ) in Fig. 6(d). The units are embedded in the arc frame to form a metasurface sample as shown in Fig. 6(e). All the used materials are poly lactic acid (PLA) plastic. In the experiment, a waveguide system $(1.3 \mathrm{~m} \times 1.3 \mathrm{~m} \times 32 \mathrm{~mm})$ is constructed to scan the reflected acoustic fields [see Fig. 6(h)]. The metasurface sample is placed in one boundary and is clamped closely by two parallel plexiglass plates $(1.5 \mathrm{~m} \times 2 \mathrm{~m} \times 6 \mathrm{~mm})$. A row of twenty-seven loudspeakers [ABS-221-RC, see Fig. 6(g)] are mounted in a box baffle by a space of $5 \mathrm{~cm}$ and surrounded with the porous foam, then placed at the opposite side far enough away from the metasurface $(1.3 \mathrm{~m})$. The absorbing wedge porous foam is installed at the other two boundaries of the experimental system to mimic an anechoic environment.

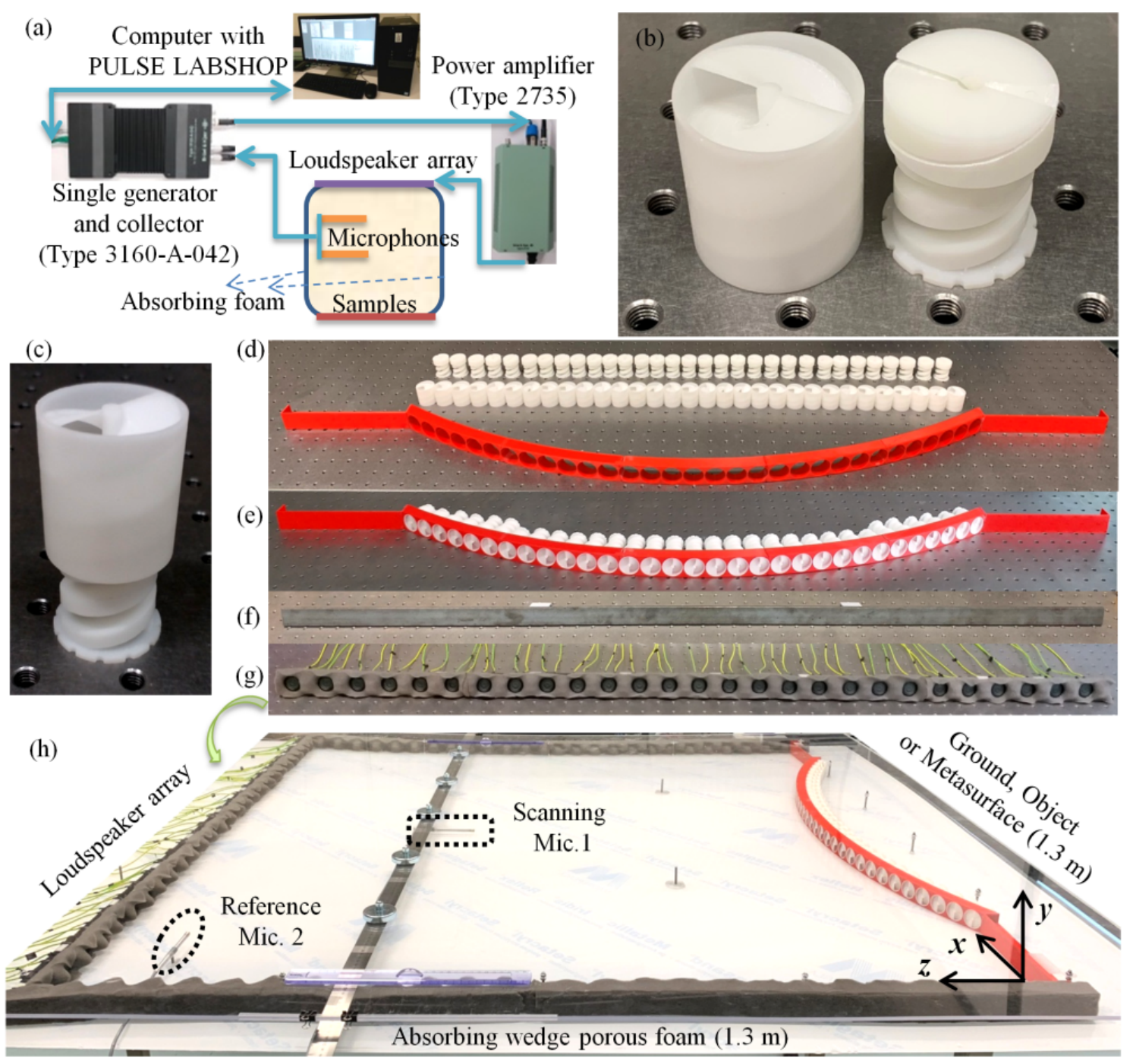

FIG. 6. The details of the experimental setup. (a) Schematic diagram of the measurement. Photographs of the fabricated helical channel and matched screw (b), the cylindrical unit sample (c), the manufactured components of 
the 31 units and the surface arc-shaped frame (d), the assembled metasurface (e), the steel sheet to imitate a flat ground (f), the loudspeaker array (g) and the experimental waveguide system (h).

Regarding the measurement process, we first use a computer with the software PULSE LABSHOP to generate a continuous sound wave single with a center frequency of $f=2.2 \mathrm{kHz}$ with $6.4 \mathrm{kHz}$ span, as well as a center frequency of $6.1 \mathrm{kHz}$ with $12.8 \mathrm{kHz}$ span, respectively. Then, the acoustic signal is modulated by a power amplifier module (Brüel \& Kjær type 2735) and emitted by the loudspeakers array. Between the loudspeakers and metasurface, two microphones (Brüel \& Kjær type 4138) are placed at designated positions to sense the local sound signals. Mic. 1 is mounted at a fixed position near the loudspeakers to detect as a reference signal. Mic.2 is pasted onto a ruler (length $2 \mathrm{~m}$ ) which is attached to the upper plate by a magnet moveable to scan the local pressure field. The measured signals from the microphones and the source signal are connected to the Brüel $\&$ Kjær LAN-XI data-acquisition hardware (model 3160-A-042) to communicate with the computer at the same time. By using the software PULSE LABSHOP, the real-time pressure data is obtained by gathering the two microphone signals based on the source signal. Then the amplitude and phase of the pressure fields are recorded at different spatial positions within the measured region. To reveal the invisibility effect of the carpet cloak, we measure a rectangular region (the white boxes as shown in Fig. 3) on the right of the waveguide center. For the lower frequency of $f=2.2 \mathrm{kHz}$ in Fig. 3(a), the measured region $\left(30 \times 30 \mathrm{~cm}^{2}\right)$ are meshed into 900 squares with a spacing of $1 \mathrm{~cm}$. And for the higher frequency of $f=6.1 \mathrm{kHz}$ in Fig. 3(b), the measured region is reduced to half area $\left(30 \times 15 \mathrm{~cm}^{2}\right)$ with a horizontal step $1 \mathrm{~cm}$ and vertical step $0.5 \mathrm{~cm}$ due to the decrease of wavelength. In addition, to expose the illusion effect quantitatively, we will survey the reflected pressure amplitude by measurement step $1 \mathrm{~cm}$ at a far-field observation line with a distance of $0.8 \mathrm{~m}$ to the flat ground, as the black dashed lines shown in Fig. 5.

For each measurement, we first remove the matesurface cloak and replace it with porous absorbing foam, then scan the measured region to sense the local values of amplitude $A_{0}$ and phase $\varphi_{0}$ as the reference background pressure field $P_{0}=A_{0} \exp \left(i \varphi_{0}\right)$. Next, the designed metasurface cloak is configured; and its amplitude $A_{\mathrm{t}}$ and phase $\varphi_{\mathrm{t}}$ of the total pressure field $P_{\mathrm{t}}=A_{\mathrm{t}} \exp \left(i \varphi_{\mathrm{t}}\right)$ can be recorded at different spatial positions within the measured region. Then, the reflected pressure field is obtained by subtracting two pressure fields with and without the metasurface cloak.

\section{Carpet cloak}

To better exhibit the cloaking effect, the pressure fields are measured in three situations, including the flat ground [a thin steel plate, $1.3 \mathrm{~m} \times 3 \mathrm{~cm} \times 6 \mathrm{~mm}$, see Fig. 6(f)], the uncloaked object (all matched screws are completely rotated into the helical channels) and the object with cloak (the designed configuration of the metasurface). The real part of the reflected pressure fields in measurement regions at the frequencies of $2.2 \mathrm{kHz}$ and $6.1 \mathrm{kHz}$ are shown in Figs. 7(a) and 7(b) for 
the three cases, respectively. When there is no object on the flat ground [see Figs. 7(a-i) and 7(b-i)], a nearly plane wave propagates in free space which also directly indicates that the loudspeakers array we handcrafted [see Fig. 6(g)] can produce a plane incident wave well. Once we place the uncloaked object, this free plane wave field is strongly scattered, and a clear interference pattern can be seen in Figs. 7(a-ii) and 7(b-ii). Then, when the uncloaked object is replaced by the metasurface cloak, the free field is nearly recovered and the scattered field toward the undesired both sides is cancelled, as shown in Figs. 7(a-iii) and 7(b-iii). Therefore, the curved metasurface cloak can mimic a flat plane and reduce the signature of the objects at different frequencies.

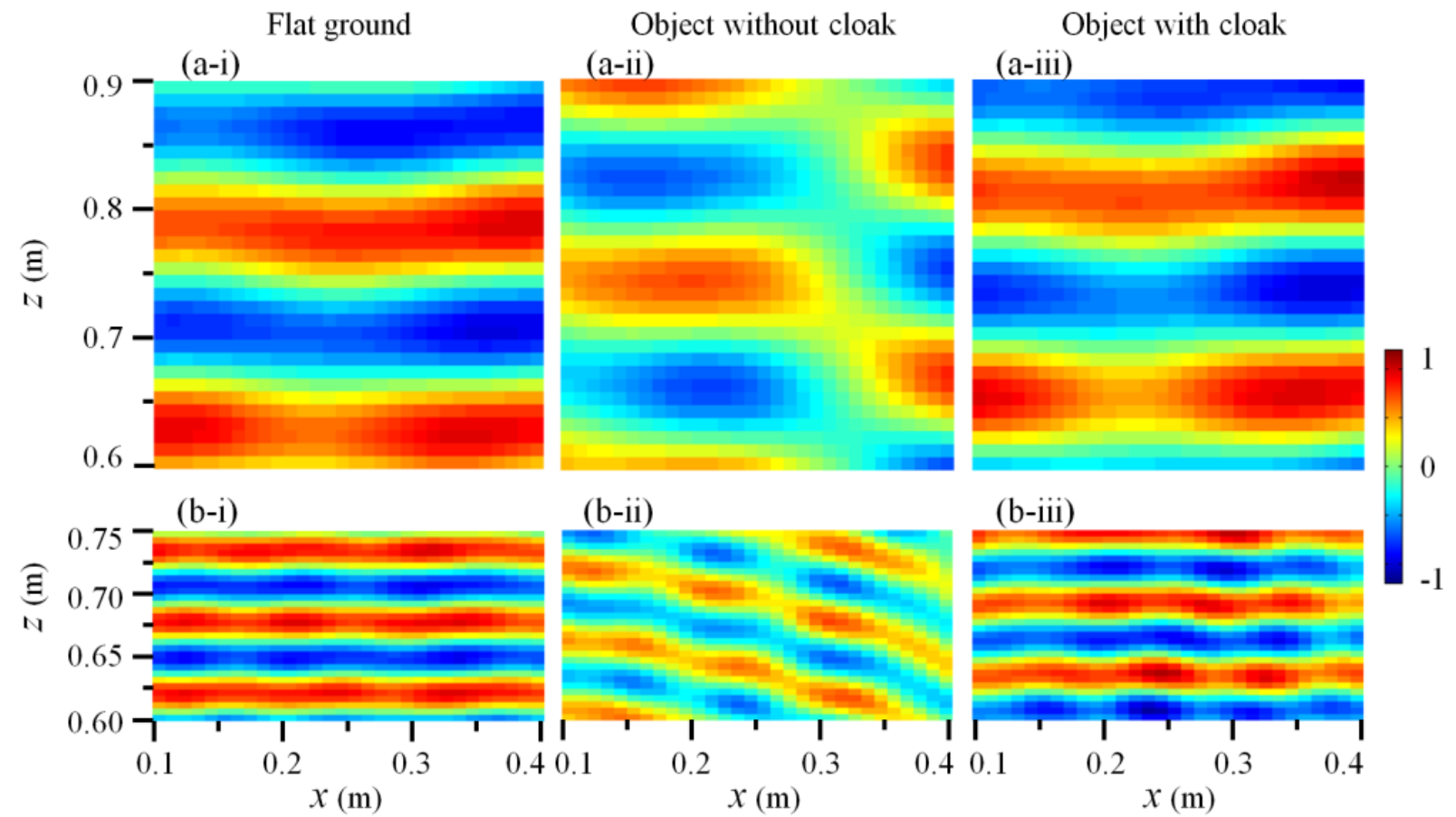

FIG. 7. Experimental measurements of the real part of the reflected acoustic field in the white dashed box regions shown in Fig. 3. The reflected sound fields of (i) the flat ground, (ii) the uncloaked object, and (iii) the object with cloak exposed to the normally impinging waves at the frequencies of $2.2 \mathrm{kHz}$ (a) and $6.1 \mathrm{kHz}$ (b).

\section{Ground illusion}

For a quantitative comparison of the ground illusion, we measure the transverse reflected amplitude at a far-field observation line with a distance of $0.8 \mathrm{~m}$ to the flat ground. The simulated and experimental results corresponding to Fig. 5 are shown in Fig. 8 at frequencies of $2.2 \mathrm{kHz}$ and $6.1 \mathrm{kHz}$ with the convex and concave ground shapes. All the results from the simulations and experiments are in good agreement with each other in terms of the number and location of the amplitude peaks, except that the values obtained from the experiments are a little lower than the ones from the simulation, especially for the illusion of the concave ground shape. Due to the inherent scattering on the convex structures and focusing on the concave one, this inevitably leads to a little bit lower reflected energy in the center of the experimental regions when we use a convex cloak to 
mimic a concave ground shape. Presumably, this slight discrepancy may also be caused by the printing error of the unit samples, the machining error of the curved surface frame and the energy loss from the air viscosity existing in the experiments. It is seen that the selected metasurface configurations can indeed guarantee a satisfactory acoustic ground illusion at a wide range of frequencies.
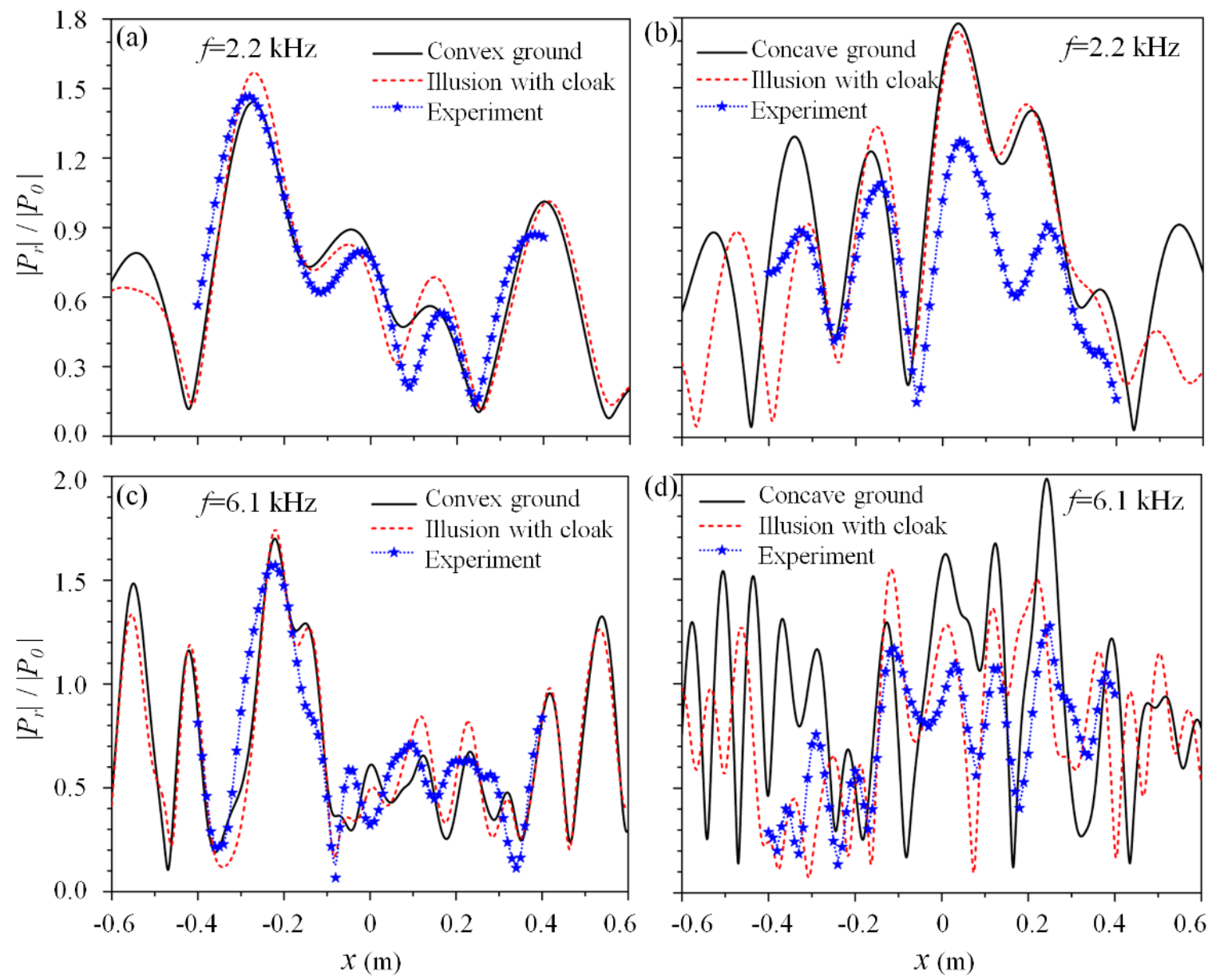

FIG. 8. The transverse reflected amplitude at a far-field observation with a distance of $0.8 \mathrm{~m}$ to the flat ground by cutting black dashed lines shown in FIG. 5. The reflected pressure amplitude of the arbitrarily curved ground exposed to the normally incident plane waves at the frequencies of $2.2 \mathrm{kHz}(\mathrm{a}-\mathrm{b})$ and $6.1 \mathrm{kHz}(\mathrm{c}-\mathrm{d})$. The illusion performances are shown in (a) and (c) with the convex ground, as well as in (b) and (d) with the concave ground.

Therefore, all results of the experimental measurements show good acoustic cloaking and illusion capabilities of the proposed continuously tunable curved metasurface in a broad frequency range.

\section{v. Conclusion}

A broadband tunable curved metasurface for acoustic cloaking and illusion has been proposed and validated theoretically, numerically and experimentally. Due to the continuously tunable matched 
screw-and-nut physical mechanism, the phase shifts cover a full $2 \pi$ span by varying the helical depth of units. The achievable subwavelength frequency range of $5 \mathrm{kHz}$ (from 2 to $7 \mathrm{kHz}$ ) is broader than those presented so far in the literatures for acoustic carpet cloaking. The proposed curved metasurface can successfully hide objects from acoustic waves by restoring the disturbed reflected field or mimicking an arbitrary shaped ground with continuous tunability. It can also be able to operate for a wide-angle detection with oblique incidence. In addition, the cloak and illusion functions at different frequencies can be achieved by just one tunable metasurface structure.

Once the corresponding phase gradient of the metasurface is designed correctly and properly, both the ground shapes and the objects behind it will become invisible. By utilizing the proposed design scheme, it should be fairly straightforward to conceal or imitate objects with different sizes and shapes, and to construct a metasurface for 3D cloaking of sound. Adopting higher spatial resolution of phase discretization may further enhance the performances of the cloak or illusion around the region with abruptly changing object or ground profiles. All these remarkable advantages bring the acoustic metasurfaces a step closer toward the realization of practical cloaks.

\section{Acknowledgements}

This work is supported by the National Natural Science Foundation of China (Grants No. 11872101 and 11991031), by la Région Grand Est, and by the Institut CARNOT ICEEL. The first two authors is grateful to the support of the National Natural Science Foundation of China (Grant No. 11502123 and 11902171), the Natural Science Foundation of Inner Mongolia Autonomous Region of China (Grant No. 2015JQ01) and China Scholarship Council (CSC Grant No. 201807090043). 


\section{Appendix A: Theoretical equivalent parameter}

It is known that the space-coiling structure can tremendously increase the inner channel length by introducing spiral passages [27, 33]. The helical channel length can be represented by an equivalent diameter at a single frequency by numerical fitting [36, 39]. Since waves at different frequencies have different wavelengths, the equivalent diameter in the spiral channel passing by the scalar sound waves is directly related to the incident frequency [43]. In order to determine the equivalent parameter $e=e(f)$ in a broad band, we define the mean deviation of the phase shift at each given frequency as

$$
P H(e)=\frac{1}{N} \sum_{n=0}^{N}\left|\phi_{\mathrm{th}}\left(e, h_{i}\right)-\phi_{\mathrm{nu}}\left(h_{i}\right)\right|,
$$

where $\phi_{\text {th }}$ is the phase shift derived from the theoretical equivalent model with the straight path and $\phi_{\mathrm{nu}}$ is the corresponding one exported from the numerical model with the spiral channel. After the numerical fitting between the straight path and the spiral channel, the equivalent parameter associated with the incident frequency can be obtained in the sub-wavelength range.
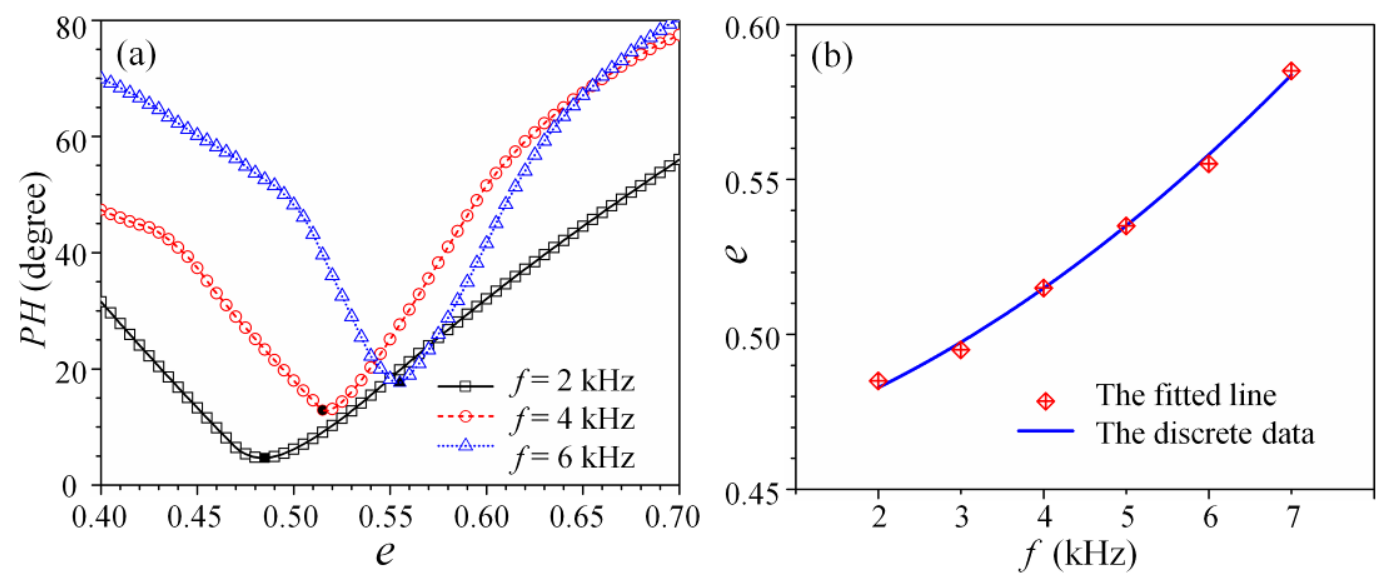

FIG. 9. The discrete and fitted results of the equivalent parameter associated with the incident frequency. (a) The mean deviation of the phase shifts varying with the equivalent parameter at different frequencies, 2, 4, 6 and $8 \mathrm{kHz}$; (b) the numerical fitting for the equivalent parameter varying with the changing frequencies.

The mean deviation of the phase shifts varying with the equivalent parameter are illustrated in Fig. 9(a) for four particular frequencies. Then, as the solid symbols shown, the minimum values of the mean deviation at different frequencies are extracted for the numerical fitting. In order to obtain the approximate expressions, the equivalent parameter are fitted by a polynomial of the 2 nd degree, which is a function of the frequency. The fitting curve is given by

$$
e=1.385 \times 10^{-9} f^{2}+7.71 \times 10^{-6} f+0.4619 \text {. }
$$

The discrete and fitted results of the equivalent parameter are plotted in Fig. 9(b). Then, in the 
tunable unit study, we can easily calculate the equivalent diameter $\left(D_{e}\right)$ and the equivalent channel length $\left(L_{e}\right)$ associated with the incident frequency using this approximate expression.

\section{Appendix B: Tuned helical depth for multiple functions}

For convenience and clarity, the corresponding tuned helical depths for the carpet cloak and the ground illusion are listed in Tables I and II. As checked and referenced values, these data may be used to tune the functions of the designed helical metasurface.

Table I. The appropriate helical depth $h_{i}(i=1,2, \ldots, 15), h_{16}$ and $h_{i}=h_{32-i}(i=17,18, \ldots, 31)(\mathrm{mm})$ corresponding to the carpet cloak at the frequencies of $f(2.2$ and $6.1 \mathrm{kHz})$ with the global incident angle $\theta_{i}^{\circ}\left(0^{\circ}, 30^{\circ}, 45^{\circ}\right.$ and $\left.60^{\circ}\right)$.

\begin{tabular}{|c|c|c|c|c|c|c|c|c|c|c|c|c|c|c|c|c|}
\hline$f, \theta_{i}^{g}$ & $h_{1}$ & $h_{2}$ & $h_{3}$ & $h_{4}$ & $h_{5}$ & $h_{6}$ & $h_{7}$ & $h_{8}$ & $h_{9}$ & $h_{10}$ & $h_{11}$ & $h_{12}$ & $h_{13}$ & $h_{14}$ & $h_{15}$ & $h_{16}$ \\
\hline $2.2 \mathrm{kHz}, 0^{\circ}$ & 8.6 & 14.6 & 16.6 & 18.2 & 20.4 & 25.6 & 7.2 & 12.7 & 14.7 & 15.8 & 16.6 & 17.1 & 17.6 & 17.9 & 18.1 & 18.2 \\
\hline $6.1 \mathrm{kHz}, 0^{\circ}$ & 5.3 & 7.2 & 15.6 & 17.4 & 25.2 & 27.1 & 9.2 & 15.6 & 16.7 & 17.8 & 21.9 & 25.4 & 26.3 & 26.6 & 26.8 & 26.9 \\
\hline $2.2 \mathrm{kHz}, 30^{\circ}$ & 7.6 & 13.9 & 16.0 & 17.4 & 18.8 & 20.7 & 24.8 & 3.6 & 10.2 & 12.9 & 14.3 & 15.1 & 15.6 & 15.9 & 16.1 & 16.1 \\
\hline $2.2 \mathrm{kHz}, 45^{\circ}$ & 6.5 & 13.0 & 15.2 & 16.4 & 17.5 & 18.5 & 19.7 & 21.4 & 24.0 & 28.4 & 3.8 & 7.6 & 9.7 & 10.9 & 11.5 & 11.7 \\
\hline $2.2 \mathrm{kHz}, 60^{\circ}$ & 4.7 & 11.1 & 13.7 & 15.0 & 15.9 & 16.6 & 17.2 & 17.7 & 18.3 & 18.8 & 19.3 & 19.7 & 20.1 & 20.5 & 20.7 & 20.8 \\
\hline $6.1 \mathrm{kHz}, 30^{\circ}$ & 5.1 & 6.8 & 13.6 & 16.5 & 18.5 & 25.7 & 27.0 & 28.3 & 23.5 & 26.1 & 26.8 & 27.2 & 27.7 & 28.3 & 28.8 & 29.0 \\
\hline $6.1 \mathrm{kHz}, 45^{\circ}$ & 4.7 & 6.4 & 8.2 & 15.3 & 16.6 & 17.8 & 23.3 & 26.1 & 26.8 & 27.4 & 28.3 & 19.8 & 22.8 & 24.5 & 25.1 & 25.2 \\
\hline $6.1 \mathrm{kHz}, 60^{\circ}$ & 4.0 & 5.9 & 6.6 & 7.8 & 12.9 & 15.6 & 16.3 & 16.9 & 17.5 & 18.5 & 20.7 & 23.5 & 24.8 & 25.4 & 25.6 & 25.7 \\
\hline
\end{tabular}

Table II. The appropriate helical depth $h_{i}(\mathrm{~mm})(i=1,2, \ldots, 31)$ corresponding to the illusion of the convex ground $(\mathrm{XG})$ or concave ground $(\mathrm{EG})$ at the frequencies of $f(2.2$ and $6.1 \mathrm{kHz})$ with the normally incident acoustic waves.

\begin{tabular}{cccccccccccccccc}
\hline$f$, ground & $h_{1}$ & \multicolumn{1}{c}{$h_{2}$} & $h_{3}$ & $h_{4}$ & $h_{5}$ & $h_{6}$ & $h_{7}$ & $h_{8}$ & $h_{9}$ & $h_{10}$ & $h_{11}$ & $h_{12}$ & $h_{13}$ & $h_{14}$ & $h_{15}$ \\
\hline $2.2 \mathrm{kHz}, \mathrm{XG}$ & 4.7 & 10.5 & 12.9 & 14.1 & 14.8 & 15.3 & 15.7 & 16.1 & 16.6 & 17 & 17.5 & 18 & 18.3 & 18.4 & 18.2 \\
$2.2 \mathrm{kHz}, \mathrm{EG}$ & 11 & 16.4 & 19.4 & 3.1 & 14.7 & 17.3 & 19.9 & 27.3 & 10.5 & 14.1 & 15.5 & 16.3 & 16.9 & 17.4 & 18 \\
$6.1 \mathrm{kHz}, \mathrm{XG}$ & 3.9 & 5.8 & 6.4 & 6.8 & 7.4 & 8.5 & 11.2 & 14.2 & 15.5 & 16.2 & 16.7 & 17.1 & 17.6 & 17.8 & 17.4 \\
$6.1 \mathrm{kHz}, \mathrm{EG}$ & 5.9 & 15.1 & 21.9 & 28.2 & 6.5 & 15.5 & 18.4 & 26.6 & 28.6 & 5.3 & 6.3 & 7 & 8.3 & 12.7 & 15.4 \\
\hline$h_{16}$ & $h_{17}$ & $h_{18}$ & $h_{19}$ & $h_{20}$ & $h_{21}$ & $h_{22}$ & $h_{23}$ & $h_{24}$ & $h_{25}$ & $h_{26}$ & $h_{27}$ & $h_{28}$ & $h_{29}$ & $h_{30}$ & $h_{31}$ \\
\hline 17.7 & 17.0 & 16.2 & 15.2 & 14.0 & 12.5 & 10.8 & 9.5 & 8.6 & 8.3 & 8.3 & 8.3 & 8.1 & 7.4 & 5.7 & 2.3 \\
18.7 & 19.6 & 20.3 & 20.8 & 20.5 & 19.5 & 18.2 & 16.7 & 14.6 & 6.0 & 20.3 & 16.9 & 12.8 & 22.0 & 17.1 & 11.9 \\
16.8 & 16.1 & 14.5 & 8.3 & 6.8 & 6.3 & 5.9 & 5.6 & 5.4 & 5.3 & 5.3 & 5.3 & 5.3 & 5.0 & 4.4 & 2.3 \\
16.3 & 16.8 & 17.3 & 17.6 & 17.4 & 16.7 & 15.6 & 7.8 & 5.8 & 27.4 & 19.6 & 14.5 & 5.6 & 26.4 & 16.2 & 6.1 \\
\hline
\end{tabular}

\section{Appendix C: Analysis for the upper limit of the operating frequency}

We implemented several full-wave simulations at high frequencies to discuss the tunable metasurface performance. The corresponding acoustic pressure fields for carpet cloaking are shown 
in Fig. 10. It can be seen that the pressure distribution at the wavefront will become distorted and uneven with the increase of the frequency in Figs. 10(a-f). As the most important parameter to evaluate the cloaking property, the reflected wavefront phase is extracted from the pressure fields at a far-field observation with a distance of $0.8 \mathrm{~m}$ from the ground. The results show that the phase fluctuates up and down within $\sim 5 \%$ and $\sim 6.6 \%$ at frequencies of $6.5 \mathrm{kHz}$ and $7 \mathrm{kHz}$, respectively, which means that the plane wavefront can be almost guaranteed, as shown in Fig. $10(\mathrm{~g})$. As the incident frequency increases above $7.5 \mathrm{kHz}$ (with the phase fluctuation range of $\sim 12.8 \%$ ), the wavefront becomes more and more confusion because of the strong diffraction effect. Therefore, the appropriate operational frequency range should be below $7 \mathrm{kHz}$.
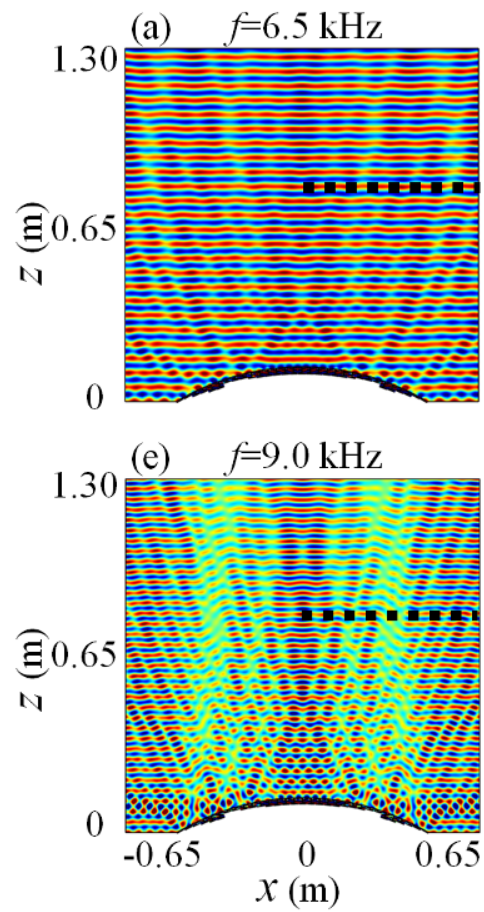
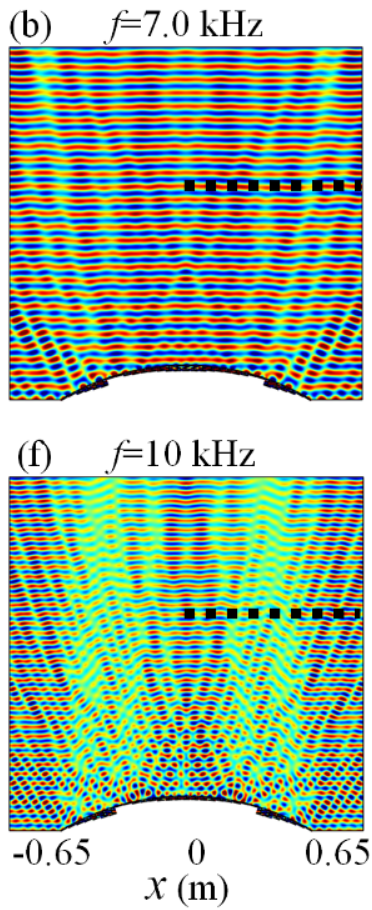
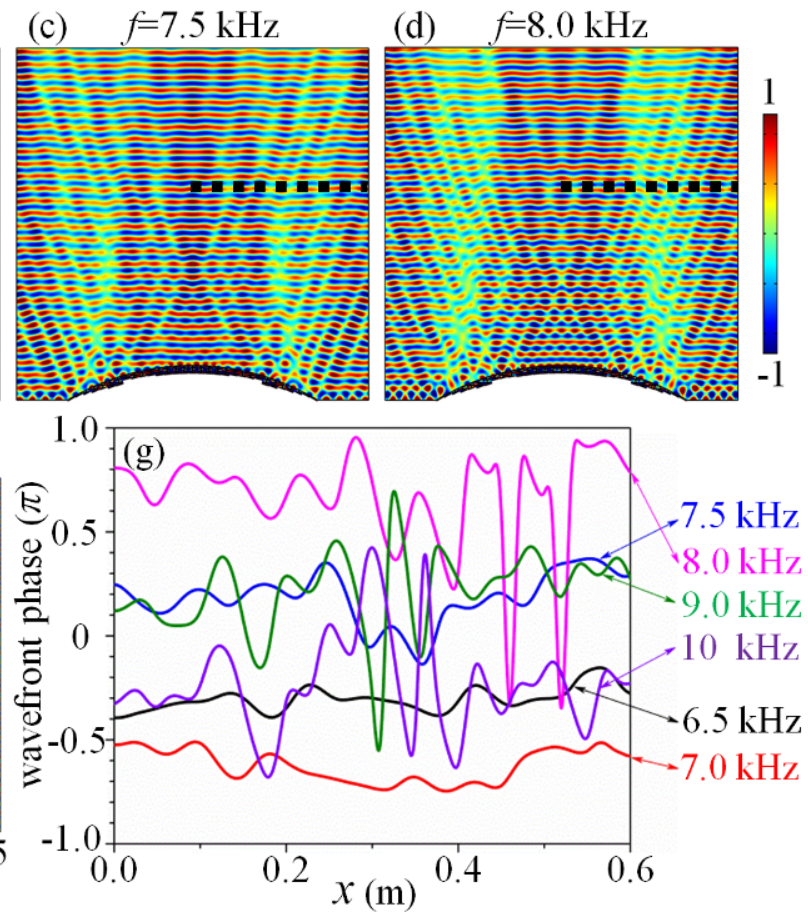

FIG. 10. Full-wave simulated scattering acoustic pressure fields for carpet cloaking at higher frequencies. The reflected fields in the $x z$-plane for the curved metasurface cloak exposed to the normally incident plane wave at the frequencies of $6.5 \mathrm{kHz}(\mathrm{a}), 7.0 \mathrm{kHz}$ (b), $7.5 \mathrm{kHz}$ (c), $8.0 \mathrm{kHz}$ (d), $9.0 \mathrm{kHz}$ (e) and $10 \mathrm{kHz}$ (f). The transverse reflected wavefront phase at a far-field observation with a distance of $0.8 \mathrm{~m}$ from the ground by cutting black dashed lines shown in (a-f).

\section{Appendix D: Influences of thermoviscous loss effects on the wave modulation of the helical unit and curved metasurface}

Thermoviscous acoustic module is employed in the helical channel region of the tunable unit with both the viscous friction and thermal diffusion being considered. The dynamic viscosity of the air in the helical channel $\eta=1.81 \times 10^{-4} \mathrm{~Pa} \cdot \mathrm{s}$, which is a high-loss case. The heat capacity at constant pressure $C_{p}=1005.4 \mathrm{~J} /(\mathrm{kg} \cdot \mathrm{K})$, and the thermal conductivity $\kappa=0.0258 \mathrm{~W} /(\mathrm{m} \cdot \mathrm{K})$. Since the channel size is in the centimeter order, the bulk viscosity can be neglected in the calculations [28, 43]. Non-slip and isothermal conditions are imposed on the solid boundaries. The pressure acoustic module is used 
for the incident and reflected areas. The acoustic-thermoviscous coupling boundary is taken into account. To guarantee calculation precision, the boundary layer properties of the mesh are set on the inner surfaces of the helical channels.
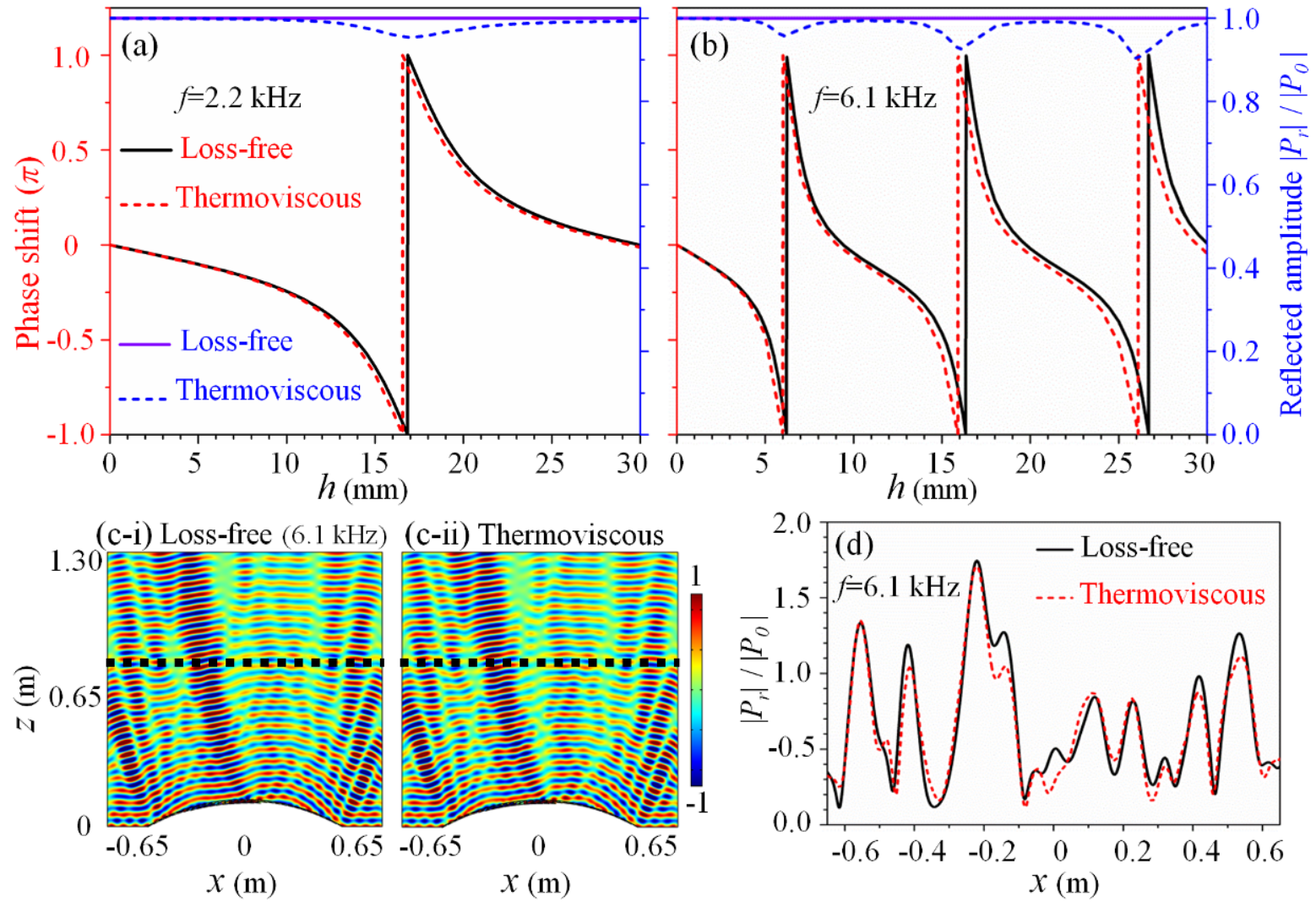

FIG. 11. Thermoviscous loss effects. The phase shift and reflected amplitude of the tunable unit with and without loss at the frequencies of $2.2 \mathrm{kHz}$ (a) and $6.1 \mathrm{kHz}$ (b). The solid and dashed lines represent the results for the loss-free and thermoviscous cases, respectively. (c) Acoustic pressure field distributions of the ground illusion at the frequency of $6.1 \mathrm{kHz}$ for (i) loss-free case and (ii) thermoviscous case. (d) The reflected pressure amplitudes along the dashed lines in (c) with the observation transverse-line locating at $z=0.8 \mathrm{~m}$.

Figs. 11(a) and (b) show the phase shift and the corresponding amplitude as functions of the unit depth of the tunable unit at different frequencies. It is observed that the thermoviscous loss slightly influence the reflected phase and amplitude responses. For the thermoviscous case, the phase shift shows a very small advance with increasing the channel length (helical depth $h$ ) because of the viscous friction and thermal dispassion, but still can cover a full $2 \pi$ region; and the thermoviscous effects result in the slight decreases of the reflected amplitude. Even in the phase jump region, the reflection amplitude is above 91\% with the maximal loss of the reflectivity. In addition, the differences for the phase and amplitude between two cases are relatively obvious at the high frequencies. In order to further illustrate the effect of thermoviscous loss on the full-wave reflection field, Fig. 11(c) shows the acoustic pressure field distributions of the ground illusion at the higher frequency of $6.1 \mathrm{kHz}$. And the reflected amplitudes along the observation transverse-line $z=0.8 \mathrm{~m}$ are 
extracted for comparison between the loss-free and thermoviscous cases. Likewise, slight decreases of the pressure amplitudes can be observed for the thermoviscous case, as shown in Fig. 11(d). Generally speaking, we do not find remarkable differences between the loss-free and thermoviscous cases. Therefore, the influences of the thermoviscous effects can be ignored in our model.

\section{Appendix E: The design and simulation for a 3D carpet cloak}

The proposed tunable metasurface can be designed for hiding an object with an arbitrary shape because of the efficient continuous phase modulation. Inspired by this $2 \mathrm{D}$ design, we further design a $3 \mathrm{D}$ cloak of a hemispherical shape, which has been concernedly by relatively few studies [9, 25, 43].
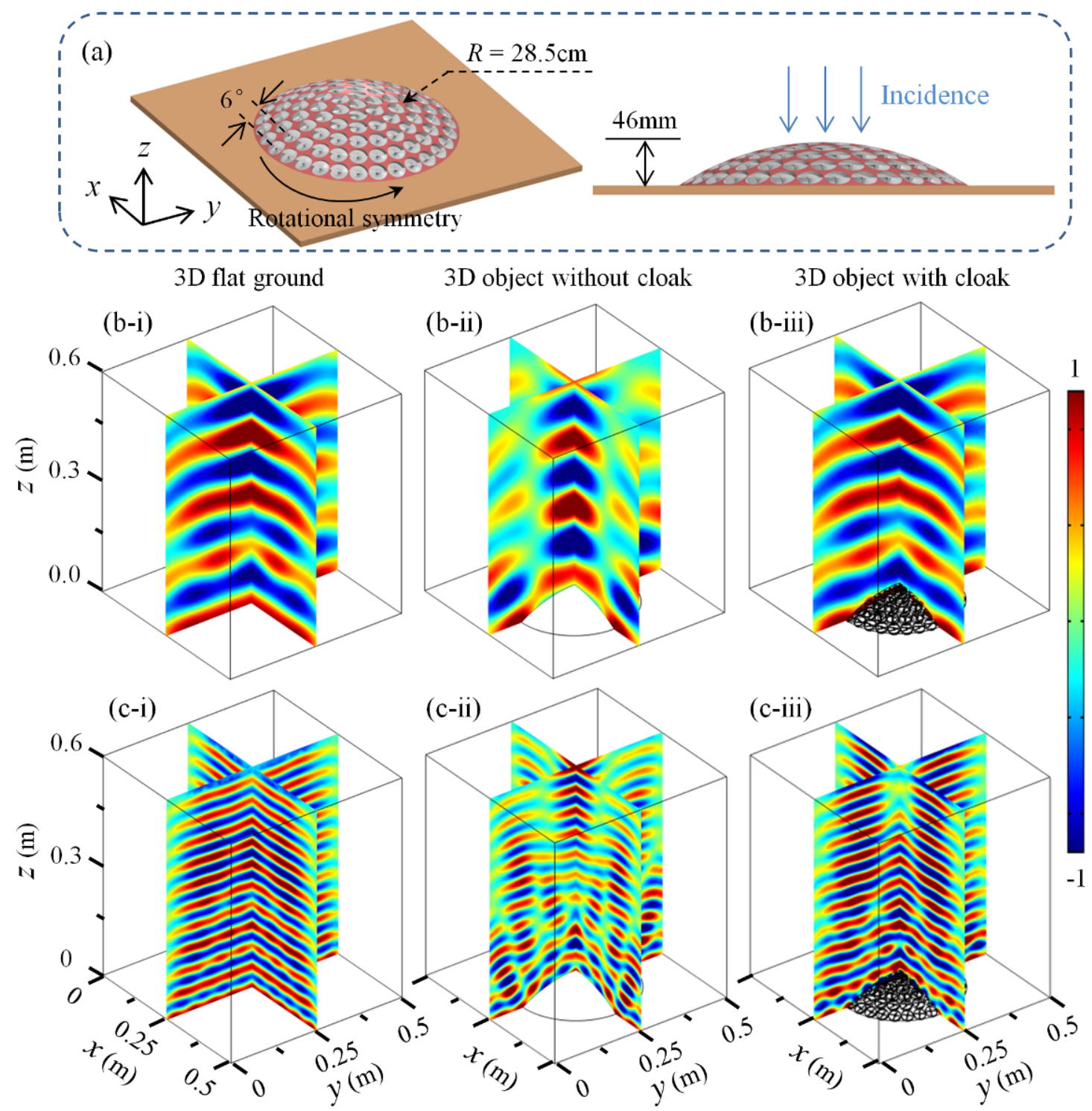

FIG. 12. Full-wave simulated scattering acoustic pressure fields for the 3D carpet cloak. (a) Schematic view of a 3D metasurface cloak and its cross section with distributed unit lattice. (b-c) The reflected fields in the $x z$ and $y z$ planes in three cases: (i) on a flat ground, (ii) on an uncloaked object (spherical-shaped bump), and (iii) on the object with the cloak exposed to the normally incident plane waves at frequencies of $2.2 \mathrm{kHz}$ (b) and $6.1 \mathrm{kHz}$ (c). 
The 3D metasurface is constructed by distributing the helical units at the pole and five latitudinal circles at intervals of $6^{\circ}$ in a hemispherical shell, as shown in Fig. 12(a). There are $6 n$ units on the $n$th $(n=1 \sim 5)$ latitudinal circles. The contour equation of the hemispherical cloak is $z(x)=\operatorname{sqrt}\left(R^{2}-x^{2}\right)-R \cos 33^{\circ}, x=R \sin \varphi$ and $\Delta \varphi=6^{\circ}$, where the radius of the sphere is $R=28.5 \mathrm{~cm}$. Then the required phase delay of each circle unit can be calculated by Eq.(4). The corresponding helical depths of the units on the latitudinal circles are obtained as $h_{i}=8.1,14.1,15.9,16.8,17.4$ and 17.6 $\mathrm{mm}$ at frequency of $f=2.2 \mathrm{kHz}$, and $h_{i}=5.2,6.9,12.9,15.9,16.5$ and $16.7 \mathrm{~mm}$ at $f=6.1 \mathrm{kHz}$, which are procured based on Figs. 2(c) and 2(d). By applying these data, Fig. 12 shows the reflected pressure fields at 2.2 and $6.1 \mathrm{kHz}$ for the cases of the flat ground, the uncloaked hemispherical object, and the object with the hemispherical cloak. The hemispherical object induces the strong acoustic scattering, resulting in a distinguished difference between the field patterns of Fig. 12(i) and (ii). With the aid of the phase delay provided by the metasurface, the scattering from the cloak is efficiently suppressed and thus the acoustic field is restored, as shown in Fig. 12(iii). The consistent field distributions with spatial plane wavefront in Figs. 12(i) and (iii) at different frequencies in Figs. 12(b) and (c) confirm the tunable cloaking effect for the 3D hemispherical object.

\section{Appendix F: The conceptual design of self-feedback automatic control for a programmable acoustic metasurface by using the real-time tunable helical unit}

Real-time steering of acoustic waves in a broadband range is one of the key functions required for acoustic cloak and illusion. The different phase compensation is required at different incoming angles and incident wave frequencies. In order to gradually steer acoustic waves in medical diagnostics and nondestructive evaluation $[45,46]$, previously, such modulation was mainly realized by expensive phased array systems (PAS) that require complex time/phase delay of each transducer. Although metasurfaces based on the phase array have been demonstrated for manipulating waves, they still have serious limitations in dynamic control of the acoustic propagation, which is a crucial feature required for real-world applications. 


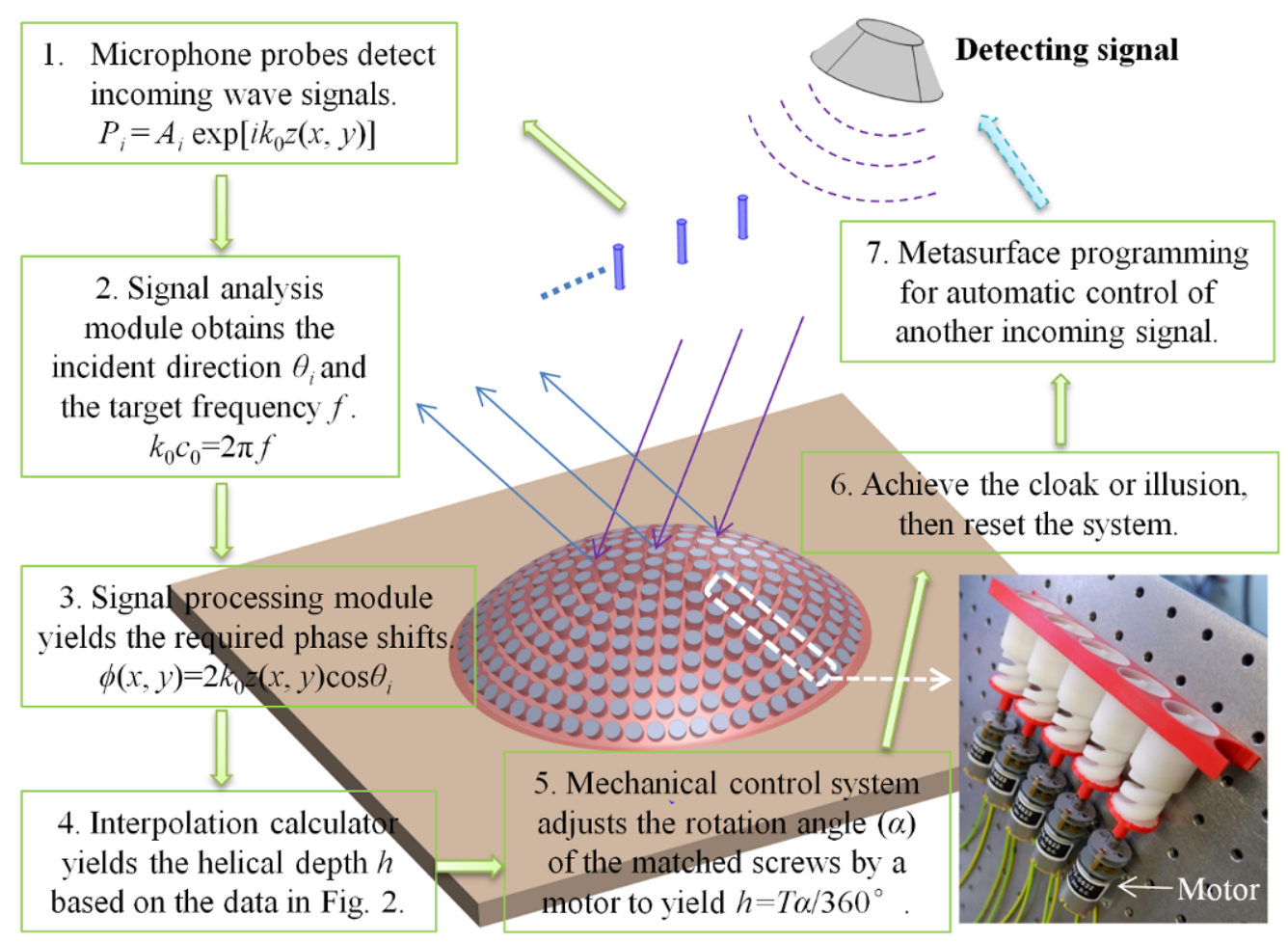

FIG. 13. A schematic representation of self-feedback real-time tunable mechanisms for the programmable

acoustic metasurface. The illustration at the bottom right shows the structure connective components of the tunable broadband mechanical control system.

Here, we propose a conceptual design of a programmable self-feedback automatic control acoustic metasurface by using our tunable helical unit, as shown in Fig. 13. When the microphone probe array detects an incoming signal, the signal analysis module can obtain the incident direction $\theta_{i}$ and target frequency $f$, then the signal processing module yields the required phase shifts $\phi$. We can set an interpolation calculator based on all the data in Fig. 2 and Eq. (4) to get the needed helical depth $h_{i}$. The helical depth of each unit can be easily controlled by adjusting the rotation angle $(\alpha)$ of the matched screws to yield $h=T \alpha / 360^{\circ}$ using a robust rotating motor. The mechanical control system is the key to achieve the automatic regulation. Its partial details are shown in the illustration at the bottom right. The proposed tunable units (white cylinders), 3D printed connectors (red gears) and small stepping motors are assembled into an arbitrarily shaped frame (red perforated plate) to fabricate a continuously and dynamically tunable cloak. Then the developed tunable system can achieve the carpet cloak or ground illusion, and be reset again to program for controlling another incident signal. This merit of the conceptual design lays the foundation for automatically manipulating acoustic waves using our helical metasurface. 


\section{References}

[1] D. Schurig, J. J. Mock, B. J. Justice, S. A. Cummer, J. B. Pendry, A. F. Starr, and D. R. Smith, Metamaterial electromagnetic cloak at microwave frequencies, Science 314, 977 (2006).

[2] M. Farhat, S. Enoch, S. Guenneau, and A. B. Movchan, Broadband cylindrical acoustic cloak for linear surface waves in a fluid, Phys. Rev. Lett. 101, 134501 (2008).

[3] S. Zhang, C. G. Xia, and N. Fang, Broadband acoustic cloak for ultrasound waves, Phys. Rev. Lett. 106, 024301 (2011).

[4] M. Farhat, S. Guenneau, and S. Enoch, Ultrabroadband elastic cloaking in thin plates, Phys. Rev. Lett. 103, 024301 (2009).

[5] N. Stenger, M. Wilhelm, and M. Wegener, Experiments on elastic cloaking in thin plates, Phys. Rev. Lett. 108, 014301 (2012).

[6] S. Brûlé, E. H. Javelaud, S. Enoch, and S. Guenneau, Experiments on seismic metamaterials: molding surface waves, Phys. Rev. Lett. 112, 133901 (2014).

[7] J. Li and J. B. Pendry, Hiding under the carpet: a new strategy for cloaking, Phys. Rev. Lett. 101, 203901 (2008).

[8] B. I. Popa, L. Zigoneanu, and S. A. Cummer, Experimental acoustic ground cloak in air, Phys. Rev. Lett. 106, 253901 (2011).

[9] L. Zigoneanu, B. I. Popa, and S. A. Cummer, Three-dimensional broadband omnidirectional acoustic ground cloak, Nat. Mater. 13, 352 (2014).

[10] N. F. Yu, P. Genevet, M. Kats, F. Aieta, J. Tetienne, F. Capasso, and Z. Gaburro, Light propagation with phase discontinuities: generalized laws of reflection and refraction, Science 334, 333 (2011).

[11] B. Assouar, B. Liang, Y. Wu, Y. Li, J. C. Cheng, and Y. Jing, Acoustic metasurfaces, Nat. Rev. Mater. 3, 460 (2018).

[12] Y. Q. Liu, Z. X. Liang, F. Liu, O. Diba, A. Lamb, and J. Li, Source illusion devices for flexural Lamb waves using elastic metasurfaces, Phys. Rev. Lett. 119, 034301 (2017).

[13] L. Y. Cao, Z. C. Yang, Y. L. Xu, and B. Assouar, Deflecting flexural wave with high transmission by using pillared elastic metasurface, Smart Mater. Struct. 27, 075051 (2018).

[14] L. Y. Cao, Y. L. Xu, B. Assouar, and Z. C. Yang, Asymmetric flexural wave transmission based on dual-layer elastic gradient metasurfaces, Appl. Phys. Lett. 113, 183506 (2018).

[15] B. Orazbayev, N. M. Estakhri, M. Beruete, and A. Alù, Terahertz carpet cloak based on a ring resonator metasurface, Phys. Rev. B 91, 195444 (2015).

[16] X. J. Ni, Z. J. Wong, M. Mrejen, Y. Wang, and X. Zhang, An ultrathin invisibility skin cloak for visible light, Science 349, 1310 (2015).

[17] S. R. Biswas, C. E. Gutiérrez, A. Nemilentsau, I. Lee, S. Oh, P. Avouris, and T. Low, Tunable graphene metasurface reflectarray for cloaking, illusion and focusing, Phys. Rev. Appl. 9, 034021 (2018)

[18] Y. Y. Yang, H. P. Wang, F. X. Yu, Z. W. Xu, and H. S. Chen, A metasurface carpet cloak for electromagnetic, 
acoustic and water waves, Sci. Rep. 6, 20219 (2016).

[19] C. Faure, O. Richoux, S. Félix, and V. Pagneux, Experiments on metasurface carpet cloaking for audible acoustics, Appl. Phys. Lett. 108, 064103 (2016).

[20] M. Dubois, C. Z. Shi, Y. Wang, and X. Zhang, A thin and conformal metasurface for illusion acoustics of rapidly changing profiles, Appl. Phys. Lett. 110, 151902 (2017).

[21] S. L. Zhai, H. J. Chen, C. L. Ding, L. L. Li, F. L. Shen, C. R. Luo, and X. P. Zhao, Ultrathin skin cloaks with metasurfaces for audible sound, J. Phys. D: Appl. Phys. 49, 225302 (2016).

[22] H. Esfahlani, S. Karkar, H. Lissek, and J. R. Mosig, Acoustic carpet cloak based on an ultrathin metasurface, Phys. Rev. B 94, 014302 (2016).

[23] F. Y. Ma, M. Huang, Y. C. Xu, J. H. Wu, Bilayer synergetic coupling double negative acoustic metasurface and cloak, Sci. Rep. 8, 5906 (2018).

[24] X. Wang, D. X. Mao, and Y. Li, Broadband acoustic skin cloak based on spiral metasurfaces, Sci. Rep. 7, 11604 (2017).

[25] Y. B. Jin, X. S. Fang, Y. Li, and D. Torrent, Engineered diffraction gratings for acoustic cloaking, Phys. Rev. Appl. 11, 011004 (2019).

[26] Y. B. Xie, W. Q. Wang, H. Y. Chen, A. Konneker, B. I. Popa, and S. A. Cummer, Wavefront modulation and subwavelength diffractive acoustics with an acoustic metasurface, Nat. Commun. 5, 5553 (2014).

[27] Z. X. Liang and J. Li, Extreme acoustic metamaterial by coiling up space, Phys. Rev. Lett. 108, 114301 (2012).

[28] Y. Li, C. Shen, Y. B. Xie, J. F. Li, W. Q. Wang, S. A. Cummer, and Y. Jing, Tunable asymmetric transmission via lossy acoustic metasurfaces, Phys. Rev. Lett. 119, 035501 (2017).

[29] B. Y. Liu and Y. Y. Jiang, Controllable asymmetric transmission via gap-tunable acoustic metasurface, Appl. Phys. Lett. 112, 173503 (2018).

[30] J. P. Xia, X. T. Zhang, H. X. Sun, S. Q. Yuan, J. Qian, and Y. Ge, Broadband tunable acoustic asymmetric focusing lens from dual-layer metasurfaces, Phys. Rev. Appl. 10, 014016 (2018).

[31] G. C. Ma, X. Y. Fan, P. Sheng, and M. Finka, Shaping reverberating sound fields with an actively tunable metasurface, Proc. Natl. Acad. Sci. USA 115, 6638 (2018).

[32] Z. H. Tian, C. Shen, J. F. Li, E. Reit, Y. Y. Gu, H. Fu, S. Cummer, and T. J. Huang, Programmable acoustic metasurfaces. Adv. Funct. Mater. 1808489 (2019).

[33] S. D. Zhao, A L. Chen, Y. S. Wang, and C. Z. Zhang, A continuously tunable acoustic metasurface for transmitted wavefront modulation, Phys. Rev. Appl. 10, 054066 (2018).

[34] A L. Chen, Q. Y. Tang, H.Y. Wang, S. D. Zhao and Y. S. WANG, Multifunction switching by a flat structurally tunable acoustic metasurface for transmitted waves, SCIENCE CHINA Physics, Mechanics \& Astronomy, doi: 10.1007/s11433-019-1498-2

[35] S. W. Fan, S. D. Zhao, A L. Chen,Y. F. Wang, B. Assouar, and Y. S. Wang, Tunable broadband reflective acoustic metasurface, Phys. Rev. Appl. 11, 044038 (2019). 
[36] X. F. Zhu, K. Li, P. Zhang, J. Zhu, J. T. Zhang, C. Tian, and S. C. Liu, Implementation of dispersion-free slow acoustic wave propagation and phase engineering with helical-structured metamaterials, Nat. Commun. 7, 11731 (2016).

[37] Y. H. Ding, E. C. Statharas, K. Yao, and M. H. Hong, A broadband acoustic metamaterial with impedance matching layer of gradient index, Appl. Phys. Lett. 110, 241903 (2017).

[38] H. Esfahlani, H. Lissek, and J. Mosig, Generation of acoustic helical wavefronts using metasurfaces, Phys. Rev. B 95, 024312 (2017).

[39] S. J. Liang, T. Liu, F. Chen, and J. Zhu, Theoretical and experimental study of gradient-helicoid acoustic metamaterial, J. Sound Vib. 442, 482 (2019).

[40] X. S. Fang, X. Wang, and Y. Li, Acoustic splitting and bending with compact coding metasurfaces, Phys. Rev. Appl. 11, 064033 (2019).

[41] N. M. Estakhri, A. Alu, Ultra-thin unidirectional carpet cloak and wavefront reconstruction with graded metasurfaces, IEEE Antennas Wireless. Propag. Lett. 13, 1775 (2014).

[42] L. Y. Hsu, T. Lepetit, and B. Kante, Extremely thin dielectric metasurface for carpet cloaking, Prog. Electromag. Res. 152, 33 (2015).

[43] L. Kinsler, Fundamentals of Acoustic, Wiley, New York, (1982).

[44] W. Q. Ji, Q. Wei, X. F. Zhu, and D. J. Wu, 3D acoustic metasurface carpet cloak based on groove structure units, J. Phys. D: Appl. Phys. 52, 325302 (2019).

[45] E. Macé, G. Montaldo, I. Cohen, M. Baulac, M. Fink, M. Tanter, Functional ultrasound imaging of the brain, Nat. Methods, 8, 662 (2011).

[46] L. Y. Yu, and Z. H. Tian, Guided wave phased array beamforming and imaging in composite plates, Ultrasonics 68, 43 (2016). 